\title{
THE ASTERIX SPECTROMETER AT LEAR
}

\author{
(ASTERIX Collaboration)
}

S. Ahmad ${ }^{1 \mathrm{a}}$, C. Amsler ${ }^{2}$, R. Armenteros ${ }^{3}$, E.G. Auld ${ }^{4}$, D.A. Axen ${ }^{4}$, D. Bailey ${ }^{3 b}$, S. Barlag ${ }^{3 c}$, G.A. Beer ${ }^{5}$, J.C. Bizot ${ }^{1}$, M. Botlo ${ }^{6 \mathrm{~d}}$, J. Butler ${ }^{3 \mathrm{e}}$, M. Comyn $^{7}$, W. Dahme ${ }^{8 \mathrm{f}}$, B. Delcourt ${ }^{1}$, M. Doser ${ }^{2 g}$, K.D. Duch ${ }^{9}$, K.L. Erdman4, F. Feld-Dahme ${ }^{8}$, U. Gastaldi ${ }^{3}$, M. Heel ${ }^{\text {9h }}$, B. Howard ${ }^{4}$, R. Howard ${ }^{4}$, J. Jeanjean ${ }^{1}$, H. Kalinowsky ${ }^{9}$, F. Kayser ${ }^{9 \mathrm{i}}$, E. Klempt ${ }^{9}$, C. Laa ${ }^{6 \mathrm{j}}$, R. Landua ${ }^{3}$, G.M. Marshall ${ }^{5}$, B. May ${ }^{9}$, H. Nguyen ${ }^{1}$, N. Prevot ${ }^{1 \mathrm{k}}$, J. Riediberger ${ }^{2}$, L.P. Robertson ${ }^{6}$, C. Sabev*, U. Schaefer ${ }^{81}$, O. Schreiber ${ }^{9 m}$, U. Straumann ${ }^{2}$, P. Truöl ${ }^{2}$, H. Vonach ${ }^{6}$, P. Weidenauer ${ }^{9}$, B.L. White ${ }^{4}$, R.W. Wodrich ${ }^{9 \mathrm{n}}$ and M. Ziegler ${ }^{90}$

1) LAL, Orsay, France.

2) University of Zurich, Switzerland.

3) CERN, Geneva, Switzerland.

4) University of British Columbia, Vancouver, BC, Canada.

5) University of Victoria, BC, Canada.

6) University of Vienna, Austria.

7) TRIUMF, Vancouver, BC, Canada.

8) University of Munich, Fed. Rep. Germany.

9) University of Mainz, Fed. Rep. Germany.

(Submitted to Nuclear Instruments and Methods in Physics Research)

Present addresses:

a) TRIUMF, Vancouver, BC, Canada.

b) University of Toronto, Ontario, Canada.

c) Max-Planck-Institut, Munich, Fed. Rep. Germany.

d) Austrian Academy of Science, Vienna, Austria.

e) Fermilab, Batavia, Ill., USA.

f) LeCroy Research Systems, Geneva, Switzerland.

g) KEK, Tsukuba, Ibaraki 305, Japan.

h) Boehringer, Ingelheim, Fed. Rep. Germany. i) Volkshochschule Hanau, Fed. Rep. Germany.

j) Vöest Alpine, Vienna, Austria.

k) Salomon, Annecy, France.

l) DVFLR, Stuttgart, Fed. Rep. Germany.

m) Siemens, Munich, Fed. Rep. Germany.

n) Schott Glaswerke, Mainz, Fed. Rep. Germany.

o) AEG, Darmstadt, Fed. Rep. Germany.

*) Visitor at CERN. 


\section{INTRODUCTION}

The ASTERIX (Antiproton STop ExpeRiment on Initial X-rays) experiment was proposed [1] in order

- to study the dynamics of $\bar{p}$ annihilation at rest, in particular the dependence on the orbital angular momentum $\mathrm{L}$ and the isospin $\mathrm{I}$ of the initial $\bar{p} p$ atomic state using $\mathrm{L} \mathrm{X}$-ray detection as a tag for P-wave annihilation;

- to study production and decay of light mesons ( $\mathrm{m} \leq 1.7 \mathrm{GeV} / \mathrm{c}^{2}$ ) with high statistics to clarify some of the open questions in hadron spectroscopy;

- to search for possible exotic (non- $\overline{\mathrm{q}} q$ ) states (glueballs, hybrids, four-quark states, $\overline{\mathrm{B} B}$ bound states);

- to observe K X-ray transitions to the ground state of the $\bar{p}$ atom and to measure its shift and width.

Before ASTERIX, most of the information on $\bar{p} p$ and $\bar{p} d$ annihilation at rest was obtained in experiments stopping $\bar{p}$ beams in liquid-hydrogen and deuterium bubble chambers [2]. Their results helped to provide the basis of the SU(3) quark model and, more recently, of QCD. Whilst the focus of high-energy hadronic physics moved to the frontiers of charm and beauty production, many questions in the low-energy region between 1 and $2 \mathrm{GeV}$ remained unanswered. Among other reasons, this was due to limitations of the bubble-chamber technique, in particular to the low statistics (about 100,000 events studied in pionic and 20,000 in kaonic channels), the lack of selective triggers, the limited separation between charged pions and kaons, and the missing-gamma detection.

With the commissioning of the Low-Energy Antiproton Ring (LEAR) [3] at CERN, with its pure, intense and monochromatic antiproton beam, the study of $\bar{p} p$ interactions at low energies with new and more advanced techniques was resumed. As the low beam momenta obtainable from LEAR made it possible to stop all the antiprotons in a gaseous hydrogen target, the basic idea of the ASTERIX experiment was to measure $\vec{p} \mathrm{p}$ annihilations with and without $\mathrm{X}$-rays from the $\overline{\mathrm{p} p}$ atomic cascade in coincidence. In a liquid-hydrogen target, annihilation occurs mainly from atomic S-states owing to the Day-Snow-Sucher mechanism [4]. In gas, the collisional Stark mixing is reduced and the $\bar{p} p$ atom annihilates more often from atomic $P$ states. A previous experiment [5] stopped antiprotons in a gaseous hydrogen target at 4 atm. It observed $\mathrm{L} X$-ray transitions into the $2 \mathrm{P}$ level with a yield of $(6 \pm 3) \%$ and established a lower limit of $90 \%$ for the ratio of the annihilation width to the radiative width of the $2 \mathrm{P}$ level. This result suggested that $\mathrm{L} \mathrm{X}$-rays signal almost pure $\mathrm{P}$-wave annihilation. The ASTERIX detector had to be capable of triggering on selected final-state topologies. The high data rates obtainable with a modern apparatus, and the available computing power of mainframe computers, made possible an increase of statistics by a factor of 10 to 100 with respect to the bubble-chamber experiments.

The physics programme required four major tasks to be performed in the set-up phase of the experiment:

i) the construction and test of a large-acceptance and low-energy threshold X-ray Drift Chamber (XDC) [6] surrounding a cylindrical $\mathrm{H}_{2}$ gas target at NTP to measure the X-rays from $\overline{\mathrm{p} p}$ atomic transitions, to give accurate tracking near the annihilation vertex, and to measure the $\mathrm{dE} / \mathrm{dx}$ of charged kaons and pions;

ii) the upgrading of a magnetic spectrometer (the former DM1 [7] at LAL, Orsay) with about 50\% solid-angle coverage, and the addition of a position sensitive gamma detector with $75 \%$ solid-angle coverage;

iii) the set-up of a flexible trigger system allowing selection of events on the basis of information on the initial state and on the topology of the final state of the annihilation;

iv) the development of software used for off-line reconstruction, final-state identification, and partial-wave analysis adapted to this detector. 
This paper gives a detailed description of the spectrometer, the trigger and data-acquisition system, the calibration and alignment procedures, the off-line software and data processing, and the main performances of the detector. Finally, it summarizes the operation over the different run periods between 1983 and 1986. A more detailed presentation of the XDC central detector will be given in a forthcoming paper.

\section{SPECTROMETER}

\subsection{General description}

The general layout of the spectrometer is shown in fig. 1. The detection volume consisted of a cylindrical region of length $1.4 \mathrm{~m}$ and internal diameter $1.8 \mathrm{~m}$, in which a homogeneous axial magnetic field of up to $0.82 \mathrm{~T}$ was produced by the extended DM1 solenoid. The original DM1 magnet had to be extended from $1.1 \mathrm{~m}$ to $1.4 \mathrm{~m}$ by adding two sets of coils and additional iron in the yoke to allow for the installation of the two end-cap gamma detectors. The magnetic field within the detection volume had a maximum deviation of $+1.4 \%$ in the radial and $-0.5 \%$ in the axial direction, compared with its value at the centre.

Antiprotons entered the solenoid along the field axis and were stopped in the cylindrical gas target, positioned in the centre of the magnet. Upstream $\left(T_{1}, T_{2}, T_{3}\right)$ and downstream $\left(T_{4}\right)$ scintillation counters were used to define the antiproton stop signal. The gas target was surrounded by a system of detectors consisting of

- an X-ray Drift Chamber (XDC);

- seven cylindrical multiwire proportional chambers (MWPCs), labelled $C_{1}, C_{2}, Q_{1}, Q_{2}, P_{1}, Q_{3}$, and $\mathrm{P}_{2}$;

- a cylindrical lead sheet after $P_{1}$ to convert gammas before $Q_{3}$;

- two position-sensitive gamma detectors (PSGD1 and PSGD2) in the end-caps of the magnet.

The detector assembly consisted of three self-supporting modules with cylindrical symmetry, the two end-cap detectors, and the beam counters. The central module included the gas target, the scintillation counter $T_{4}$, and the XDC. Chambers $C_{1}$ and $C_{2}$ constituted the second module. The third module was the large assembly of chambers $\mathrm{Q}_{1}, \mathrm{Q}_{2}, \mathrm{P}_{1}, \mathrm{Q}_{3}$, and $\mathrm{P}_{2}$. A lead converter, 0.9 radiation length thick, was positioned between $\mathrm{P}_{1}$ and $\mathrm{Q}_{3}$ in order to achieve, together with the two end-cap detectors, a coverage of $75 \%$ solid angle for $\gamma$-ray conversion.

The electronic system used for the XDC readout was the one developed for the UA1 central detector [8]. Additional gap-time modules, developed for this experiment [9], were added to trigger on X-rays. The MWPC readout system was based on CERN receiver-memory hybrid (RMH) modules [10]. Some of the modules were modified to allow for the use of the digitized readout signals in the trigger system based on programmable random access memories (RAMs). The control of the event recording, the electronics readout, and the data transfer to tape was done by a PDP-11/60 computer using the standard CERN data-acquisition system [11]. Monitoring, control, and on-line display of events were handled by three SUPERCAVIAR - small stand-alone microcomputers developed at CERN [12] - or, at a later stage, by a VAX-750.

\subsection{Antiproton beam and hydrogen target}

The LEAR machine delivered antiproton beams in the years 1983 to 1986 with a rate of up to $10^{6}$ antiprotons per second in a continuous mode at selected momenta between $105 \mathrm{MeV} / \mathrm{c}$ and $1600 \mathrm{MeV} / \mathrm{c}$, with a momentum spread $\delta \mathrm{p} / \mathrm{p} \approx 10^{-3}$. Since the ASTERIX experiment was designed to stop antiprotons in a $\mathrm{H}_{2}$ gas target, data were taken at the lowest available beam momenta, which were $300 \mathrm{MeV} / \mathrm{c}$ in $1983,200 \mathrm{MeV} / \mathrm{c}$ in 1984 , and $105 \mathrm{MeV} / \mathrm{c}$ in 1985 and 1986 . Typical intensities used for data taking were $10^{4}-10^{5}$ antiprotons per second. 
The arrangement of the beam-defining plastic scintillators and of the XDC is shown in fig. 2. A retractable MPWC was mounted in the beam line in front of the beam scintillation counters to monitor the size of the antiproton beam in both the horizontal and the vertical plane. Incoming antiprotons first fired the $T_{1}$ scintillator. Just before the entrance to the $\mathrm{H}_{2}$ gas target there was a $50 \mu \mathrm{m}$ thick plastic scintillator $\left(\mathrm{T}_{2}\right)$, which was surrounded by a cylindrical scintillator $\left(\mathrm{T}_{3}\right)$. The assembly of the beam-defining scintillators $T_{2}$ and $T_{3}$, with their light-guides but without light-screens, is shown in fig. 3. The $T_{3}$ counter detected and vetoed charged particles from events with antiprotons annihilating in $T_{1}$ or $T_{2}$ or the target entrance window. At the opposite end of the gas target there was a $25 \mathrm{~mm}$ thick, $130 \mathrm{~mm}$ diameter scintillator $\left(\mathrm{T}_{4}\right)$, which was used to veto antiprotons passing through the target without annihilating. The $T_{1}$ scintillator was removed when the antiproton momentum was reduced to $105 \mathrm{MeV} / \mathrm{c}$. An antiproton stop was defined by coincident pulses from $T_{1}$ and $T_{2}$ (or $T_{2}$ alone at $105 \mathrm{MeV} / \mathrm{c}$ ) and no pulses from either $T_{3}$ or $T_{4}$. The $T_{2}$ signal defined the start time of the event.

At $105 \mathrm{MeV} / \mathrm{c}$, there was a variable air gap $(1.6$ to $3 \mathrm{~cm}$ ) between the beam pipe and the target window, and less than $100 \mu \mathrm{m}$ of dense material (scintillator, its light reflectors, and a very thin Mylar moderator) between the end of the beam pipe and the target, enabling the antiprotons to enter. More than $99 \%$ of all antiprotons stopped inside the target. Figure 4 shows a plot of the annihilation vertex distribution in the target, derived from the reconstruction of multiprong events. At higher momenta, a moderator was placed upstream of $T_{1}$, the thickness of the moderator being adjusted to maximize the antiproton stopping rate in the target.

The size of the $\mathrm{H}_{2}$ gas target was a compromise between the width of the stopping distribution for various beam momenta and the desire to have charged-particle tracking in the XDC close to the annihilation point so as to optimize the vertex determination. A diameter of $160 \mathrm{~mm}$ and a length of $760 \mathrm{~mm}$ (between $\mathrm{T}_{2}$ and $\mathrm{T}_{4}$ ) was chosen.

\subsection{The X-ray drift chamber}

The XDC [6] was a cylindrical projection chamber with radial electric field (fig. 5). It surrounded the $\mathrm{H}_{2}$ gas target, from which it was separated by a $6 \mu \mathrm{m}$ thick aluminized Mylar foil, at $-10 \mathrm{kV}$ voltage, acting as the cathode. Ninety sense wires (Ni-Cr, $32 \mu \mathrm{m}$ diameter, $1.5 \mathrm{k} \Omega / \mathrm{m}$ ) at ground potential and 270 field wires ( $\mathrm{Cu}-\mathrm{Be}, 100 \mu \mathrm{m}$ diameter) at typical potentials between -2.1 and $-2.2 \mathrm{kV}$ were stretched between two flanges held by an aluminium external tube $(0.5 \mathrm{~mm}$ thick, $340 \mathrm{~mm}$ internal diameter). This tube provided the mechanical rigidity for the entire structure.

The XDC was continuously flushed with a gas mixture of argon $(50 \%)+$ ethane $(50 \%)$. Likewise, the $\mathrm{H}_{2}$ gas target was flushed to minimize possible argon contamination from the XDC gas diffusing through the Mylar foil.

A drift cell in the XDC was defined by five field wires and one sense wire. With the magnetic field on, the drift cells of the electrons were curved (see fig. 5). At $0.8 \mathrm{~T}$, the maximum drift angle was $0.30 \mathrm{rad}$ and the drift velocity $5 \mathrm{~cm} / \mu \mathrm{s}$, leading to a maximum drift time of $1.3 \mu \mathrm{s}$. This particular gas mixture was chosen in order to minimize the dependence of the drift velocity on the electric field. The drift velocity in the XDC was constant within $\pm 10 \%$.

The XDC electronics was the same as the UA1 central detector electronics [8], with additional modules built at the University of Mainz [9] to trigger on X-ray candidates. Charge-sensitive preamplifiers at each end of the 90 signal wires allowed z-position measurements by charge division. The signals were fed via twisted-pair cables into eight charge-time digitizers (CTDs) where, after additional amplification, the left signal and the sum of the signals, integrated over $32 \mathrm{~ns}$, were digitized in 6-bit flash analog-to-digital converters (FADCs). The FADC response was non-linear to extend its dynamical range. The digitized values were continuously written into 90 memory banks with a depth of $128 \times 12$ bits ( 6 bits for energy, 6 bits for z-position), until a stop signal interrupted 
the process. The memories were then read out by three readout processors (ROPs), allowing access to all information from the last $4 \mu \mathrm{s}$. A time interpolator indicated, with an accuracy of $\pm 2 \mathrm{~ns}$, the arrival time of the electrons produced at the boundary of a drift cell and giving a signal exceeding an adjustable threshold.

The following information about each pulse detected in the XDC was obtained from the recorded data:

- the total charge as a function of time (in $32 \mathrm{~ns}, 6$-bit amplitude samples),

- the mean z-position (6-bit) of each $32 \mathrm{~ns}$ sample,

- the drift-time associated with the leading edge of each pulse, with $\pm 2 \mathrm{~ns}$ accuracy (10-bit).

The distinction between signals from X-rays and charged particles was achieved by using the difference between their ionization properties. X-rays convert within a volume of less than $1 \mathrm{~mm}^{3}$, and the primary electrons usually stay within one drift cell of the XDC, firing only one signal wire. On the other hand, charged particles ionize along their path, crossing typically four drift cells, so that several neighbouring signal wires are fired. This is illustrated in fig. 6. A further distinction between $\mathrm{X}$-rays and charged particles is the fast decaying pulse for X-rays, compared with the approximately constant pulses from charged particles.

\subsection{The multiwire proportional chambers}

Seven cylindrical MWPCs (see table 1) were installed inside the DM1 magnet. Five chambers $\left(C_{1}, C_{2}, Q_{1}, Q_{2}\right.$, and $\left.Q_{3}\right)$ had both anode and cathode readout, giving the $r, \phi$, and $z$ coordinates of the charged-particle hits, whilst the remaining two chambers $\left(P_{1}, P_{2}\right)$ had only anode readout, giving the $\mathrm{r}$ and $\phi$ coordinates only.

The construction details of $\mathrm{C}_{1}$ and $\mathrm{C}_{2}$, built at Carleton University, Ottawa, are described in ref. [13]. The structural cylinders were made of $6.35 \mathrm{~mm}$ Hexcell (nylon fibre, phenolic resin honeycombs). The anode gold-plated tungsten wires, $20 \mu \mathrm{m}$ in diameter, were stretched parallel to the cylinder axis. The cathode planes were made from $0.11 \mathrm{~mm}$ thick aluminized Mylar. The aluminium was deposited in $2 \mathrm{~mm}$ wide strips at angles of $\pm 35^{\circ}$ (with respect to the anode wires) for the inner and outer cathode planes of $C_{1}$, and of $\pm 45^{\circ}$ for those of $C_{2}$. Chambers $C_{1}$ and $C_{2}$ were operated at a nominal voltage of $3.4 \mathrm{kV}$ and reached efficiencies greater than $98 \%$ at antiproton stop rates of up to $3 \times 10^{4}$ antiprotons per second.

Chambers $Q_{1}, Q_{2}$, and $Q_{3}$ [7] were previously used in the DM1 detector at Orsay. The cathode planes were formed of $50 \mu \mathrm{m}$ diameter Mo wires wound helically, at approximately $45^{\circ}$ with respect to the anode plane, on a support composed of $250 \mu \mathrm{m}$ diameter fibre-glass threads. The $30 \mu \mathrm{m}$ diameter tungsten anode wires had $2 \mathrm{~mm}$ spacing and were strung parallel to the axis. The chambers were made gas-tight by means of $25 \mu \mathrm{m}$ Mylar sheets stretched between the end rings. The efficiencies of the Q chambers - operated at $4.3 \mathrm{kV}$ - were about $95 \%$ for the anodes and $85 \%$ for the cathodes at $3 \times 10^{4}$ antiprotons per second.

The structural cylindrical cathode planes of $P_{1}$ and $P_{2}$ [14] were made of a sandwich of $10 \mathrm{~mm}$ thick Rohacell and $50 \mu \mathrm{m}$ thick aluminium foil. Gold-plated tungsten anode wires of $20 \mu \mathrm{m}$ diameter were strung with a $2 \mathrm{~mm}$ spacing parallel to the chamber axis. Both chambers had a detection efficiency close to $97 \%$ at $3.0 \mathrm{kV}$.

The total thickness of the seven MWPCs and the XDC was 0.016 radiation length. All MWPCs used a gas mixture of argon $(68.4 \%)+$ isobutane $(28 \%)+$ methylal $(3.4 \%)+$ Freon $(0.2 \%)$.

\subsection{The Gamma Detectors}

Gamma rays were detected by their conversion in $6 \mathrm{~mm}$ lead foils ( 0.9 radiation length thick). A cylindrical converter foil was inserted between chambers $P_{1}$ and $Q_{3}$ (1567 $\mathrm{mm}$ diameter), and the 
forward and backward regions were covered by two end-cap position-sensitive gamma detectors (PSGD1 and PSGD2).

For the end-cap detectors (fig. 7), the design was chosen such that it would optimize the position resolution for medium-energy photons in order to reconstruct channels with single photons, $\pi^{0}$ 's and $\eta$ 's. Measurements in a $100 \mathrm{MeV}$ tagged photon beam, and shower code simulations, indicated that optimum spatial resolution was obtainable if a one radiation length converter is immediately followed by a MWPC.

Each end-cap detector consisted of three hexagonal chambers, each with two cathode-strip planes and an anode wire plane. The anode and cathode wires ran parallel to the sides of the hexagon, i.e. $0^{\circ}$ and $\pm 60^{\circ}$, respectively. Thus a total of nine coordinates could be measured within the detector thickness of $75 \mathrm{~mm}$. The anode-cathode gap was $5 \mathrm{~mm}$, the wire spacing $4 \mathrm{~mm}$, and the strip width $3.8 \mathrm{~mm}$, with $0.2 \mathrm{~mm}$ spacing between the strips. The cathode strips consisted of $35 \mu \mathrm{m}$ $\mathrm{Cu}$ on $75 \mu \mathrm{m}$ Kapton foils. Honeycomb $(10 \mathrm{~mm})$, sealed with two $0.1 \mathrm{~mm}$ thick foils of fibre-glass reinforced epoxy on each side, provided support for the cathode foils. The sandwich was glued to a typically $75 \mathrm{~mm}$ wide, hexagonal, fibre-glass reinforced epoxy frame, having an outer diameter of $1663 \mathrm{~mm}$. This frame made the whole assembly self-supporting and allowed for an active chamber surface of $1.3 \mathrm{~m}^{2}$ after subtraction of the centre frame area, which was necessary in order to let the scintillator light-guides and the antiprotons enter the spectrometer. Table 2 summarizes the parameters of the detector.

The $5 \mathrm{~mm}$ thick hard lead $[\mathrm{Pb}(\mathrm{Sn})]$ converter, laminated with $4 \mathrm{~mm}$ thick fibre-glass reinforced epoxy sheets, was contained in the second cathode plane of the first chamber. All anodes and cathodes following this plane were read out, providing an effective resolution of $\pm 2 \mathrm{~mm}$ for the anodes and the cathodes. To veto charged particles in the first chamber before the converter, the anode wire and cathode strip readouts were grouped in five wires or five strips, respectively, to save on electronics costs.

The chamber performance was monitored between runs, using cosmic muons. With $2500 \mathrm{~V}$ anode voltage, we reached efficiencies greater than $98 \%$ on all planes except the cathode planes close to the lead foil, which reached only $80 \%$ efficiency.

\section{TRIGGER}

\subsection{General description}

The trigger system was designed to select $\vec{p} p$ annihilation events with different initial and final states. Apart from these main triggers, triggers for calibrating the XDC with radioactive sources were also taken, as well as alignment triggers with cosmic rays and electronic test triggers to check the 23,722 MWPC readout channels.

To combine these various requirements in one hardware set-up, a programmable system based on commercial ECL-technology CAMAC modules was used. A three-level trigger scheme was employed for implementing the slow-trigger processors for sophisticated on-line filtering, still keeping the dead-time as short as possible. The programmability was realized by extensive use of RAMs as coincidence and logic decision units. This gave great flexibility in changing trigger conditions without hardware modifications, resulting in a user-friendly trigger set-up procedure and avoiding mistakes due to errors in cabling, timing, etc. Since the contents of the trigger RAMs were written to tape at the beginning of each run, the trigger actually used was always well documented. Typical decision times were

- $400 \mathrm{~ns}$ for a $\overline{\mathrm{p}}$ Stop signal;

- $800 \mathrm{~ns}$ for the charged particle multiplicity;

- $1.6 \mu \mathrm{s}$ for the detection of X-rays; 
- $150 \mu \mathrm{s}$ to $1 \mathrm{~ms}$ for the reconstruction of four charged tracks and the calculation of their transverse momenta;

- 6 to $8 \mathrm{~ms}$ for the rejection of $\overline{\mathrm{p}}$ stops in the argon of the XDC, including the reset of the readout processor for long events producing an overflow in the event buffer.

The trigger layout is shown in fig. 8. Analog signals from the beam scintillation counters, from the cosmic-ray scintillation counters mounted below the magnet yoke, and from test pulsers were fed into different discriminators with programmable thresholds for dealing with the various signal levels. The output signals were fed into RAM units running as programmable overlap coincidence units in which the proper trigger definition could be selected. A second stage of RAM was used as shapers, and two programmable output lines were combined in a fast $O R$ to build the main timing signal for the subsequent units. The status of all triggers was recorded in a trigger register. The main timing signal latched the hit information from the MWPCs in the RMH receiver modules and started a time gate for the XDC X-ray trigger logic, which covered the maximum possible drift-time inside the central detector. This gate was closed after $4 \mu \mathrm{s}$ and the trigger information was latched into RAM Level 1.

After $4 \mu$ s following a $\overline{\mathrm{p}}$ stop, a first decision, based on the charged-particle multiplicity and the presence of an X-ray candidate, was taken in the logical decision unit RAM Level 1: either to keep the event, or to reset the full system through a system reset. At $1.5 \mu \mathrm{s}$ after the reset decision the apparatus was ready to accept the next event by opening the gate of the trigger register. If the event was accepted, the RAM Level 1 started the readout of the XDC and RMH information in two separate processors. These two processors provided information on antiprotons stopping in the counter gas, and on the missing mass or missing momentum of the charged-particle final state in the transverse plane. The use of these two processors was optional, and for the major part of the data taking, events were accepted using only decisions on trigger-level 1 after about $4 \mu \mathrm{s}$.

If the two processors were included in the active trigger, a RAM unit Level 2 combined the trigger information and made the final decision whether to keep the event and interrupt the data-acquisition computer for a complete readout, or to perform a system reset. This decision was made 1 to $5 \mathrm{~ms}$ after the main timing signal, depending on the amount of XDC information. After an event was accepted, all the trigger status information was stored on tape for off-line analysis.

Thirty-two ECL scalers in a CAMAC module were continuously monitoring rates inside the trigger logic (e.g. single scintillation counter rates, event rejection rates on different trigger levels, beam intensity, trigger dead-time) to control the stability of the apparatus.

The full trigger set-up information (contents of all RAMs, thresholds, timings) was written on tape before each run so as to keep track of the selected trigger condition. A set of programs was developed for implementing new trigger conditions, testing the set-up, and storing the various trigger versions on disk, whence they could be accessed from menus during the run initialization phase.

\subsection{XDC triggers}

The XDC X-ray trigger logic checked for an X-ray detected during a period of $1.4 \mu \mathrm{s}$ after a $\overline{\mathrm{p}}$ stop by looking for an isolated hit on one sense wire with no hits on the two neighbouring wires. This logic consisted of eight 'one-gap' modules monitoring the discriminator output of each sense wire via the CTD modules. They had an X-ray flag register which could be read remotely, and a flag line was set by the system if an X-ray candidate was present.

The XDC data were passed, via REMUS readout [15], from the readout processors in the CTD crates to an auxiliary crate controller (ACC). The ACC searched for unwanted events with large energy deposits on one or more sense wires, due to a $\overline{\mathrm{p}}$ stop in the XDC counter gas, and for events with very large buffer lengths which were produced mainly by low-momentum particles spiralling in the central detector. 


\subsection{MWPC triggers}

The number of tracks from the annihilation was determined by a fast multiplicity logic unit which tested the number of clustered hits in four of the seven MWPCs $\left(C_{1}, C_{2}, Q_{2}, P_{1}\right)$ using the memory OR output of the RMH receiver modules. The logic consisted of ECL-based CAMAC majority logic modules, which gave an analog signal corresponding to the number of clustered hits input to the module. Fast ADCs digitized this information in each plane, and a RAM module was used to define the number of tracks required. To allow for detection inefficiencies and noise hits in the chamber, the RAM logic could be programmed also to accept for each chamber a multiplicity interval around a preselected number. An accept flag was set if the trigger conditions were fulfilled.

A high-speed hardware processor type GA103, developed at Orsay [16], read the anode information of the MWPCs via an RMH branch splitter, using the re-read capability of the RMH system. It reconstructed tracks in the (transverse) $\mathrm{r}-\phi$ plane of the detector and calculated the transverse momentum of tracks reaching $\mathrm{Q}_{3}$ within $200 \mu \mathrm{s}$, using arithmetic boards and look-up tables. Thus it was possible to trigger on reaction channels with low branching ratios, such as $\overline{\mathrm{p}} \mathrm{p} \rightarrow \pi^{+} \pi^{-} \eta$, by setting a window on the missing mass in the transverse plane. Status lines indicated if the trigger condition was fulfilled.

\section{CHAMBER READOUT, DATA ACQUISITION, AND MONITORING}

The data-acquisition system was based on a PDP-11/60 computer in connection with a GEC-Elliott (Fisher Controls) CAMAC system crate which was supported by the CERN data-acquisition software programs. To achieve the highest possible data collection rate, the control of the spectrometer and the on-line display of events and histograms was performed by three separate SUPERCAVIAR [12] microcomputers, which had access, via CAMAC, to the control parameters of the apparatus and to events from the main data stream. A CERNET computer link transferred a sample of the data to the CERN IBM mainframe computer for an initial analysis during a run, in order to check the overall performance of the apparatus. In 1985 and 1986, a VAX-750 computer was used for the control, on-line analysis, and display tasks. The typical readout time for a four-prong event in the spectrometer is shown in table 3. The typical event size was $0.8 \mathrm{kB}$ for two-prong and $1.4 \mathrm{kB}$ for four-prong events. The header of each event record contained the trigger condition under which that event had been selected. The data were written on high-density ( $6250 \mathrm{bpi}$ ) magnetic tapes for later analysis, and they were transferred in blocks of about 10 events. The tape-writing overhead led to a maximum event data-taking rate of 50-70 events per second. Figure 9 shows a schematic view of the data-acquisition system.

\subsection{XDC readout}

The information from the XDC was first processed by three readout processors (ROPs) - developed at CERN [8] - which compacted the data coming from the different charge and time digitizer modules in each of the three crates, as shown in fig. 10. The 128 memory-cell contents for each wire were scanned for pulse height, position, and time information, and the ROP then reduced this information to a hit buffer, combining all information belonging to a pulse on a wire. These hit buffers were collected in a REMUS [15] compatible output buffer and were read out via a standard REMUS branch driver by an ACC (CES Firecracker model ACC 2160). Its output buffer was transferred via direct memory access (DMA) to the PDP-11/60 data-acquisition (DAQ) computer.

\subsection{MWPC readout}

The seven MWPCs and the two PSGDs had 23,722 electronic readout channels. A system of $29 \mathrm{RMH}$ crates with $480 \mathrm{RMH}$ receiver modules [10] was used to store the three-dimensional readout 
information (fig. 11). The RMH preamplifiers located close to the detector were connected to the chambers by $4 \mathrm{~m}$ shielded strip lines. These preamplifiers were then connected to the RMH receiver modules in the counting room by 400 ns delay cables. A standard RMH system (based on ECL circuitry, developed at CERN) with a cluster renormalization unit scanned the receiver modules for valid data. The event information was stored in a CAMAC buffer to be read by the DAQ computer via DMA. Majority logic units were used to sample the memory OR outputs, available from the receiver modules, in order to provide hard-wired multiplicity information for the trigger system.

The cathode signals from the three chambers $Q_{1}, Q_{2}$, and $Q_{3}$ were processed in a separate readout system built at Orsay (fig. 12). The 200 high-gain receiver modules were grouped together in 12 sector crates, each containing 96 wires of $Q_{1}, 160$ wires of $Q_{2}$, and 192 wires of $Q_{3}$. These crates were read by a hardware controller, which scanned the receiver for valid data, performed a cluster calculation, and filled an output buffer in an RMH compatible format. This output buffer was then accessed by the DAQ computer via a CAMAC interface, in a manner similar to that of the standard RMH system.

A CAMAC-based test system was used to send test pulses into preselected preamplifiers of the RMH system to test the proper operation of the complete electronic readout chain in the set-up phase and before run periods.

\subsection{On-line display and control}

A set-up of three SUPERCAVIARS was used to display sampled events on-line to monitor the spectrometer performance during a run. One SUPERCAVIAR acted as a master device (Master SUPERCAVIAR). It transferred data buffers to and from the DAQ computer via a SUPERCAVIAR-CAMAC interface. Sampled event buffers were transmitted from the Master SUPERCAVIAR to the Slave SUPERCAVIAR 1 over an advanced data-link control (ADLC) loop. This Slave SUPERCAVIAR histogrammed the hit wire distributions, timing distributions, etc., of raw data events. The contents of these histograms were displayed on a video monitor in the control room. A number of events were processed by an event reconstruction program allowing an on-line event display on a colour video monitor. The operator controlled the Master SUPERCAVIAR by means of a touch panel, which allowed direct access to control parameters of the apparatus. The Slave SUPERCAVIAR 2 was connected to two CAMAC crates in which several analog-to-digital devices collected the control parameters of the spectrometer (e.g. magnetic field, temperature inside the magnet, gas flow in the chambers, high-voltage distribution). The status of these parameters was continually monitored and displayed in the control room. In 1985 and 1986, all these tasks were transferred to a VAX-750 computer.

\section{CALIBRATION AND ALIGNMENT}

\subsection{XDC}

The XDC was calibrated with X-rays from a ${ }^{54} \mathrm{Mn}(5.5 \mathrm{keV})$ source permanently installed on the $\mathrm{T}_{4}$ counter, and from a ${ }^{57} \mathrm{Co}$ source $(6.5$ and $14.4 \mathrm{keV})$ installed on a scintillator rod positioned on the target axis in beam 'off' conditions. The X-rays were used to determine the energy resolution and the overall response of the system, including amplification at the wire and in the preamplifiers, the FADC response, and its linearization function. The energy resolution was $25 \%$ FWHM at $5.5 \mathrm{keV}$ and scaled with $\mathrm{E}^{-1 / 2}$ at lower energies. At higher energies, the resolution remained constant because of the non-linear response function of the 6-bit FADC. The energy calibration was known with an accuracy of $\pm 1.4 \%$, or better, below $10 \mathrm{keV}$. Figures $13 \mathrm{a}$ and $13 \mathrm{~b}$ show the calibration lines from ${ }^{54} \mathrm{Mn}$ and ${ }^{57} \mathrm{Co}$, respectively, demonstrating that the XDC and its associated electronics covered the full range of interest for the spectroscopy of $\bar{p} p$ atoms. 
The in-beam calibration was provided by the $\mathrm{L}_{\alpha}$ line of the $\overline{\mathrm{p} p}$ atom $(\mathrm{E}=1.734 \mathrm{keV})$ - which is singled out of the L-line pattern by requiring $\mathrm{X}$-rays in coincidence from the $\mathrm{M}$ series (fig. 13c) - and the $5.5 \mathrm{keV}$ line of the ${ }^{54} \mathrm{Mn}$ source. During the run period, this source was screened to illuminate only the downstream part of the 90 sense wires.

In the presence of a magnetic field, the drift cells of the XDC were curved, and a charged particle traversing the XDC crossed, on the average, 4 of the 90 drift cells (fig. 14). Since the charge collection first occurs from the region near the cell boundaries, the position of a charged particle passing through the XDC can be determined very precisely from the drift-time of the leading pulse edge. The 4 ns uncertainty in the drift-time measurement corresponds to $100 \mu \mathrm{m}$ spatial resolution, which is deteriorated, mainly by longitudinal diffusion, to an accuracy of $400 \mu \mathrm{m}$. The calibration of the drift-time table - correlating the measured drift-time and wire number with a point in the $x-y$ plane (at radii less than $13.5 \mathrm{~cm}$ ) - was done using collinear events from the reaction $\bar{p} p \rightarrow \pi^{+} \pi^{-}$. For these events, both tracks lie on the same circle crossing the MWPCs and the XDC, and the tracks in the XDC can be predicted with high accuracy from the MWPC points only. The drift-time table was adjusted to minimize the residuals in the XDC so as to reach the final accuracy of $\sigma=400 \mu \mathrm{m}$.

\subsection{MWPC}

The spatial position of the seven MWPCs with respect to each other was determined in two ways. Between runs, cosmic-ray events were used with the magnetic field off. The final alignment with the magnetic field on was done using again collinear events from the reaction $\bar{p} p \rightarrow \pi^{+} \pi^{-}$: since both tracks lie on the same circle of known radius $(r=3.87 \mathrm{~m}$ at $0.8 \mathrm{~T})$, two points in a reference chamber (e.g. $Q_{2}$ ) suffice for the prediction of the impact points in all other chambers in the $x-y$ and $z$ planes. The alignment constants for each chamber were determined separately by minimizing the residuals in that chamber for about $1000 \pi^{+} \pi^{-}$events.

\section{OFF-LINE PROCESSING}

\subsection{Event reconstruction}

Each event on the raw data tapes (RDT) was processed by the reconstruction program. The program output, together with a copy of the raw event, was written to the data summary tape (DST). The typical reconstruction time was $60 \mathrm{~ms}$ (IBM 370/168-3 CPU time) for a two-prong event and $170 \mathrm{~ms}$ for a four-prong event. The reconstruction program contained about 16,000 lines of FORTRAN code. Raw data were processed on IBM-3081 and IBM-3090 computers at the Landesrechenzentrum in Mainz, on an IBM-3033 at the University of Zurich, at the CERN computing centre, and on an Amdahl V8 computer at the University of British Columbia. The total CPU time consumed in the processing of about 1000 RDTs was approximately $4000 \mathrm{~h}$.

The program included

- the recognition and the fitting of charged particle tracks in the XDC and the MWPCs;

- the $\mathrm{dE} / \mathrm{dx}$ measurement in the XDC;

- the determination of energy and conversion point of X-rays;

- the determination of the position of converted gammas;

- the determination of two-track subvertices and the common annihilation vertex.

The pattern recognition was done separately for the XDC and the MWPCs. In the MWPCs, the program searched for two track segments (in $C_{1}, C_{2}, Q_{1}$ and $Q_{2}, P_{1}, Q_{3}$ ) in the transverse ( $\left.x-y\right)$ plane. If two segments could be connected, all points belonging to the two segments were submitted to a circle fit. If a good fit was obtained, the program searched for the corresponding hits in the $z$ direction, and then fitted the points to a straight line in the $\mathrm{z}-\phi$ plane $(\phi=$ angle of rotation seen from the centre of the track helix). In the XDC, each hit was positioned in space according to its drift-time and the wire number. Tracks in the $x-y$ plane were found using a road method, starting 
from hits within $5 \mathrm{~mm}$ from the sense wire. The resolution in $\mathrm{z}$ was not sufficient to determine the $\mathrm{XDC}$ track parameters also in the $\mathrm{z}-\phi$ plane. Tracks were accepted by the program if matching track segments in the XDC and the MWPCs were found, and if at most one residual was greater than a wire distance in all chambers up to $\mathrm{Q}_{2}$ (at most two in the case of $\mathrm{Q}_{3}$ ).

The track momentum in the transverse plane was determined by fitting a circle through all XDC and MWPC hits belonging to the same track. The radius of the circle is proportional to the transverse momentum, since the magnetic field is homogeneous. The longitudinal momentum was determined by fitting all cathode-wire hits in the MWPCs belonging to the same track, to a straight line in the $z-\phi$ plane ( $\phi=$ angle from the centre of the track helix to the chamber hit), and then using the slope and the transverse momentum to calculate the longitudinal momentum.

In the XDC, the ionization of a track was sampled in about 40 bins of $32 \mathrm{~ns}$. The $\mathrm{dE} / \mathrm{dx}$ was determined using the truncated mean (TM50) of the sample. Gain variations in the XDC were corrected off-line by normalizing each run to the average $\mathrm{dE} / \mathrm{dx}$ of minimum-ionizing particles.

Converted gammas were identified by hits after the lead converters, i.e. in $Q_{3}$ and $P_{2}$ or in the second and third chambers of the end-caps, and no charged track leading to these points. The conversion point was defined by the average of all hit clusters belonging to the same shower.

Every pair of tracks was combined to search for a common origin (subvertex), which could be different from the annihilation vertex (e.g. $K_{S}$ decay). Since the resolution in the $x-y$ plane was three times superior to that in the $z-\phi$ plane, the subvertex was first determined in the $x-y$ plane by the intersection of the projections of the two track helices. A subvertex was accepted if the $z$ distance of the two helices at the $x-y$ intersection point was less than $2 \mathrm{~cm}$. Each subvertex was assigned an error, taking into account the track measurement error and the intersection angle of the two tracks.

The annihilation vertex in the $x-y$ plane was defined as the point with the smallest weighted distance to all two-track subvertices. The weight was the inverse of the square of the subvertex error. The annihilation vertex in $z$ was the weighted average of the $z$ coordinates of all tracks at this $x-y$ vertex. If the separation between two subvertices exceeded three times the calculated error, a flag was set indicating a possible $\mathrm{K}_{\mathrm{S}}$ candidate.

\subsection{Monte Carlo simulation}

A simulation program was developed to

- generate any final state of $\bar{p} p$ annihilation with any given intermediate resonant state and its decay channels, assuming phase-space distribution;

- simulate the response of the detector (ionization from X-rays and charged particles, multiple scattering, decaying particles, $\gamma$ conversion);

- simulate the response of the electronics (FADC digitization, charge division, RMH readout);

- produce simulated events of identical format as real events-apart from the additional information on the initial momentum configuration - to be submitted to the same reconstruction program without modifications.

The program was used extensively to study the detector acceptance for various final states, to optimize trigger efficiencies, to provide guidance for the selection of final states, and to give the necessary input to the partial-wave analyses. The typical time to generate a two-prong event was $120 \mathrm{~ms}$ (IBM 370/168-3 CPU time), and $250 \mathrm{~ms}$ for a four-prong event.

\subsection{Kinematic fit}

A program was developed to apply a least-square fitting procedure to satisfy the constraints of energy and momentum conservation, using Lagrange multipliers to compute the corrections that must be applied to the measured momentum vectors within their errors. For $\bar{p} p \rightarrow \pi^{+} \pi^{-} \pi^{+} \pi^{-}$, there are four constraints ( $4 \mathrm{C}$ fit); if one particle of known mass (e.g. $\left.\pi^{0}\right)$ is missing, only one constraint 
(1C fit) is left. The comparison of the corrections with the measurement errors gives the probability of a chosen hypothesis. The kinematic fit program used about $30 \mathrm{~ms}$ CPU time per event and per hypothesis.

\section{PERFORMANCES}

\subsection{Initial-state selection}

Antiproton-proton annihilation at rest proceeds through the formation of a $\bar{p} p$ atom in a highly $\left(\mathrm{n} \approx 30\right.$ ) excited state. De-excitation takes place mainly by collisions with neighbouring $\mathrm{H}_{2}$ molecules, and the Stark mixing with $\mathrm{nS}$ levels [4] leads to S-wave annihilation before low-lying atomic states are reached. At lower densities, the collision rate is reduced, and the $\bar{p} \mathrm{p}$ atom has a greater chance to reach states with $\mathrm{n} \leq 5$, where radiative transitions start to dominate. The detection of an L X-ray signals the transition into the $2 \mathrm{P}$ level and occurs with a probability of $(12 \pm 2) \%$ in $\mathrm{H}_{2}$ gas at NTP [17]. The annihilation width of the $2 \mathrm{P}$ level is about 100 times greater than the radiative width for transitions into the $1 \mathrm{~S}$ ground state, hence $\mathrm{L} \mathrm{X}$-rays signal $\mathrm{P}$-wave annihilation with about $99 \%$ probability. The detection efficiency of the XDC in the $\mathrm{L}$ X-ray region $(1.74-3.11 \mathrm{keV}$ ) is $25-40 \%$ (see fig. 15 ).

However, the annihilation itself is also a source of X-rays. Charged particles in the final state are accelerated during the annihilation and give rise to inner bremsstrahlung (IBS) [18]. The IBS spectrum has a 1/E energy dependence, and its detected yield depends on the momenta of the charged particles in the final state and on the XDC detection efficiency. The IBS in the energy region 1-4 keV cannot be distinguished from $\bar{p}$ p atomic $L ~ X-r a y s$ and constitutes a background. In contrast to events with an L X-ray in coincidence (annihilating from the 2P level with $99 \%$ probability), we have no information about the initial state of annihilation for events with an IBS X-ray; the probability of $\mathrm{P}$-wave annihilation is determined only by the cascade of $\bar{p} \mathrm{p}$ atoms in $\mathrm{H}_{2}$ gas at NTP, i.e. $(50 \pm 6) \%$ [19]. Figure 16 shows an X-ray energy spectrum in coincidence with two charged particles in the final state. The majority of X-rays in the energy region above $4 \mathrm{keV}$ are due to IBS, whilst at low energies $\mathrm{L}$ and $\mathrm{M} X$-rays dominate. The IBS spectrum was calculated quantitatively and agreed very well with the observed spectrum [20]. Since the IBS yield and angular distribution

depends on the annihilation channel, it must be calculated for each channel separately. The background contribution depends on the final state and on the ratio of the annihilation rates in $\mathrm{H}_{2}$ gas and from the $2 \mathrm{P}$ state. The IBS background is typically $10-30 \%$ of the $\mathrm{L} \mathrm{X}$-ray yield; therefore an X-ray in the $1-4 \mathrm{keV}$ region signals $\mathrm{P}$-wave annihilation with about $85-95 \%$ probability. With an $\mathrm{L} X$-ray yield of $12 \%$ and an average detection efficiency of about $35 \%$, the percentage of events accompanied by an L X-ray candidate was about $4 \%$. When recording data selected for the presence of an X-ray candidate, this percentage was increased to $25-50 \%$ using the XDC one-gap trigger described in subsection 3.2 .

\subsection{Final state}

A typical reconstructed event from the on-line display with particle tracks, X-ray energy and position, and gamma-ray locations is shown in fig. 17. An enlargement of the XDC is displayed in the upper left corner of the figure, with $\mathrm{r}-\phi$ projections of the charged-particle tracks in the MWPCs. The $\mathrm{z}-\phi$ plot of the wire hits in the seven cylindrical MWPCs is displayed on the right. The squares indicate the reconstructed anode-cathode crossings. The wires triggered in the end-cap gamma detectors are shown at the lower left of the display. The reconstruction of the event shows two positively and two negatively charged prongs, accompanied by an X-ray and two gamma-rays (identified by dollar signs), one gamma-ray being converted in the cylindrical lead sheet between the $P_{1}$ and the $Q_{3}$ chamber, the second one in the downstream end-cap detector. 
The acceptance of the XDC ( $90 \%$ for charged particles) was sufficient to determine the event multiplicity. Tracks in the XDC were recognized with the algorithm mentioned in Section 6 . The acceptance of the spectrometer was $75 \%(50 \%) \times 4 \pi$ for charged particles reaching $Q_{2}\left(Q_{3}\right)$. Tracks reaching $\mathrm{Q}_{2}$ had typically 3-4 points in the MWPCs, those reaching $\mathrm{Q}_{3}$ typically 4-6 points. The pattern recognition efficiency-determined by visual scanning of several hundred events-was about $85-95 \%$. It depended strongly on the chamber anode and cathode efficiencies, the electronic noise level in the detector, and the multiplicity of the final state.

The azimuthal position resolution obtained in the XDC was $\sigma=400 \mathrm{~mm}$, and the measurement error was mainly due to longitudinal diffusion in the counter gas. The position resolution along the wire was only $\sigma=30 \mathrm{~mm}$. The azimuthal and the longitudinal position resolution of the MWPCs agreed with the expected $\sigma=600 \mu \mathrm{m}$ and $\sigma=2.2 \mathrm{~mm}$, respectively. Figure 18 shows the momentum resolution as a function of the momentum for tracks reaching $\mathrm{Q}_{3}$. It is approximated by $\sigma(\mathrm{p}) / \mathrm{p}=\left[(0.035 \mathrm{p})^{2}+(0.02)^{2}+(0.002 / \mathrm{p})^{2}\right]^{1 / 2}$, with $\mathrm{p}$ in $\mathrm{GeV} / \mathrm{c}$. Without XDC, the momentum resolution deteriorated by $30 \%$. The resolution was determined using collinear events, $\bar{p} p \rightarrow \pi^{+} \pi^{-}$ $(\mathrm{p}=927.8 \mathrm{MeV} / \mathrm{c})$, and from a comparison of Monte Carlo generated and reconstructed momenta. The quantitative agreement between Monte Carlo simulations and real data was verified by comparing the missing mass and the invariant mass resolution in different final states.

The total amount of material between the $\mathrm{H}_{2}$ target and the lead foil was 0.016 radiation length for normal incidence. The direction resolution for tracks with $p>500 \mathrm{MeV} / \mathrm{c}$ was therefore dominated by the position measurement errors and not by the multiple scattering. The distribution of the opening angle for collinear events $\bar{p} p \rightarrow \pi^{+} \pi^{-}$is shown in fig. 19. It is centred around $180^{\circ}$ and has a width $\sigma=8 \mathrm{mrad}$.

The $\mathrm{dE} / \mathrm{dx}$ of charged particles was measured in the XDC from 40 samples $(\geq 1.6 \mathrm{~mm}$ track length each) using the truncated mean (TM50) technique. Each sample was corrected for the inclination of the track with respect to the beam axis and for the track length corresponding to each time bin. A resolution $\sigma=14 \%$ for minimum-ionizing particles was obtained. Figure 20 shows the $\mathrm{dE} / \mathrm{dx}$ distribution for events $\overline{\mathrm{p}} \mathrm{p} \rightarrow \pi^{+} \pi^{-} \mathrm{K}^{+} \mathrm{K}^{-}$. Charged pions and kaons could be separated with at least $1 \sigma$ up to $400 \mathrm{MeV} / \mathrm{c}$.

The annihilation vertex was determined by extrapolating the track helices from the XDC and the MWPC into the hydrogen target, using the method described in subsection 6.1. Figure 21 shows the distance $d$ of individual tracks to the main vertex in the transverse plane, for four-prong events $(\sigma=700 \mu \mathrm{m})$. In four-prong events, the vertex in the $\mathrm{x}-\mathrm{y}$ plane is found from the weighted average of the two-track crossings. The $z$ distance of tracks to the annihilation vertex $(\sigma=2.1 \mathrm{~mm})$ is shown in fig. 22.

The detection of secondary vertices was essential for the $\mathrm{K}_{\mathrm{S}}$ identification. The average separation of two-prong crossings from the annihilation vertex shows how well $\mathrm{K}_{\mathrm{S}}$ candidates were identified by requiring a spatial separation of the decay vertex from the main vertex. The distribution has a width $\sigma=2.4 \mathrm{~mm}$ in the transverse plane (fig. 23) and $\sigma=7 \mathrm{~mm}$ in the $\mathrm{z}$ direction.

The vertex and the gamma conversion point define the gamma direction. For events with one missing particle decaying into two gamma rays (e.g. $\pi^{0}, \eta$ ), the measurement of both gamma directions was used to calculate the $2 \gamma$ invariant mass. The gamma-detection efficiency was measured using four-prong events fitting the hypothesis $\overline{\mathrm{p} p} \rightarrow \pi^{+} \pi^{-} \pi^{+} \pi^{-} \pi^{0}$ with a probability greater than $1 \%$, and having one or two detected gamma rays. Since the $\pi^{0}$ momentum is equal to the missing momentum, the direction and the energy of each gamma is defined by the conversion points. Therefore one gamma can be used to predict the impact point of the other gamma, and the ratio of the number of detected and predicted gammas gives the detection efficiency as a function of the gamma energy (fig. 24). The detection efficiency increases to a constant value of $(25 \pm 2) \%$ at $150 \mathrm{MeV}$. The measured efficiency agrees well with Monte Carlo simulations. 
The detector performance is illustrated through several missing mass, missing momentum, and invariant mass spectra in inclusive and exclusive channels for two- and four-prong events.

The momentum resolution is illustrated by the momentum spectrum for collinear events $\overline{\mathrm{p} p} \rightarrow \pi^{+} \pi^{-}$and $\mathrm{K}^{+} \mathrm{K}^{-}$. The resolution obtained by fitting both tracks separately (fig. 25a) is improved by more than a factor of 4 with both tracks fitted to a single circle (fig. 25b). The missing-mass squared recoiling against two charged pions (fig. 26a) shows clear peaks from missing $\pi^{0}, \eta$, and $\omega$, decaying into neutral or undetected particles. The missing-mass squared resolution for the inclusive $\pi^{0}$ peak is $\sigma=0.06 \mathrm{GeV}^{2}$. A large gain in resolution and a strong background reduction are obtained for two-prong events with two detected gammas (fig. 26b) by calculating the invariant $2 \gamma$ mass $\left(\sigma=0.019 \mathrm{GeV}^{2}\right)$. The missing-mass squared recoiling against four charged pions (fig. 27) displays a spike at $\mathrm{MM}^{2}=0$ due to events with no missing particles; the broader Gaussian below comes from events $\overline{\mathrm{p} p} \rightarrow \pi^{+} \pi^{-} \pi^{+} \pi^{-} \pi^{0}$. The resolution for the missing $\pi^{0}$ in four-prong events is $\sigma=0.0012 \mathrm{GeV}^{2}$. The missing energy distribution of such events is shown in fig. 28a. The peak at zero energy $(\sigma=32 \mathrm{MeV})$ is due to $\overline{\mathrm{p} p} \rightarrow \pi^{+} \pi^{-} \pi^{+} \pi^{-}$. Figure $28 \mathrm{~b}$ shows the missing momentum distribution for the same event sample $(\sigma=32 \mathrm{MeV} / \mathrm{c})$.

The invariant mass distribution of two- or three-particle resonances is illustrated with the following examples. The $\Phi$ meson, recoiling against a $\pi^{+} \pi^{-}$pair and decaying into $\mathrm{K}^{+} \mathrm{K}^{-}$, is observed (fig. 29) with a width of $\sigma=4.4 \mathrm{MeV}$. The $\pi^{+} \pi^{-} \pi^{0}$ mass-squared distribution from events $\overline{\mathrm{pp}} \rightarrow \pi^{+} \pi^{-} \pi^{+} \pi^{-} \pi^{0}$ displays the $\eta$ and the $\omega$ with a resolution $\sigma=6 \mathrm{MeV}$ and $14 \mathrm{MeV}$, respectively. The invariant mass resolution for two-body annihilations of the type $\overline{p p} \rightarrow \pi^{0} \mathrm{X}$, with $\mathrm{X} \rightarrow \pi^{+} \pi^{-}$, has been obtained from Monte Carlo simulations (fig. 31).

Table 4 summarizes the main performances of the apparatus.

\section{OPERATION}

Table 5 summarizes the different run periods, beam momenta, beam time, trigger conditions and total number of events taken in the ASTERIX experiment.

\section{CONCLUSION}

The overall hardware performance was satisfactory, although some of the older chambers suffered from inefficiency problems related to their age. The physics analysis of our data was only possible after a good quantitative understanding of the detector had been reached. This process involved several iterations in the analysis software chain (reconstruction, Monte Carlo, kinematic fit). The overall statistics increased by almost two orders of magnitude compared with bubble chamber experiments. However, several limitations of the detector reduced this gain considerably, since severe selection criteria had to be applied to get event samples of a quality comparable to that obtained from bubble chambers. These limitations were, in particular, the $50 \%$ solid angle of the detector, the small redundancy in the number of hits per track, the missing $\pi^{ \pm}, \mathrm{K}^{ \pm}$separation above $400 \mathrm{MeV} / \mathrm{c}$, and the limited gamma detection efficiency, which only allowed access to events with at most two gammas in the final state.

The ASTERIX experiment, proposed in 1980, was constructed and installed in the years 1981 to 1983, and took data from 1983 to 1986. It was dismantled and removed from the floor at the end of 1986. Several new physics results have been obtained and published:

- The first observation of K X-ray transitions into the 1S ground state of protonium [17].

- The identification of the $K_{\alpha}$ line requiring $L X$-rays in coincidence, and the measurement of the hadronic width and shift of the 1S ground state [21].

- The quantitative understanding of inner bremsstrahlung in $\bar{p} p$ annihilation and its correlation with the final state [20].

- The cascade time in hydrogen gas at NTP [22]. 
- The measurement of the annihilation branching ratio of $\pi^{+} \pi^{-}$and $\mathrm{K}^{+} \mathrm{K}^{-}$from atomic P-states, and the discovery of an increase for $\pi^{+} \pi^{-}$and a strong decrease for $\mathrm{K}^{+} \mathrm{K}^{-}$compared with S-wave annihilation [19].

- The measurement of the annihilation branching ratio of $\mathrm{K}^{0} \overline{\mathrm{K}}^{0}$ from atomic P-states, which is suppressed by a factor of 10 compared with S-wave annihilation [23].

- The strong dependence of $\pi e$ production on the initial angular momentum and isospin of the annihilation, and the increase of $\pi \mathrm{f}_{2}(1270)$ production in P-wave annihilation [24].

- The discovery of a new dipion resonance [25] with $\mathrm{I}^{\mathrm{G}} \mathrm{J}^{\mathrm{PC}}=0^{+} 2^{++}$at $\mathrm{m}=1565 \mathrm{MeV} / \mathrm{c}^{2}$ [25].

- The strong decrease of $\mathrm{E}(1420)$ production in P-waves compared with S-wave annihilation in the channel $\bar{p} p \rightarrow \pi^{+} \pi^{-} \mathrm{E}$, suggesting the $\mathrm{J}^{\mathrm{PC}}=0^{-+}$assignment for the $\mathrm{E}(1420)$ [26].

- The upper limit of $2 \times 10^{-3}$ established for narrow resonances ('baryonium') produced by $\bar{p} p$ annihilation in $\mathrm{H}_{2}$ gas by the emission of monochromatic charged pions [27].

The analysis is continuing, with emphasis on meson spectroscopy and the determination of further two- and three-body annihilation branching ratios from P-waves. Two new experiments are being installed at LEAR in 1988, continuing and extending our physics program with better detectors: i) the Crystal Barrel experiment, with particular emphasis on neutral final states, and ii) the OBELIX experiment, designed to collect high-statistics data in kaonic final states. A general overview over the physics program at LEAR in the post-ACOL era is given in ref. [28].

\section{Acknowledgements}

This work was supported by: the Bundesministerium für Forschung und Technologie; the Institut National de Physique Nuclaire et de Physique des Particules; the Schweizer Nationalfonds (in part); the Österreichische Nationalfonds; and the Natural Sciences and Engineering Research Council of Canada. We thank the LEAR team for their support during the runs and for their successful efforts to run the machine at lower and lower momenta. The contributions of M. Caria and R. Schneider in the seting-up phase of the experiment are acknowledged. We are indebted to several services and staff of the collaborating institutes for their numerous contributions. Special thanks are due to: R. Famy and M. Rebut of the CERN scintillator workshop for making the special array of scintillation counters; the CERN EP and EF support groups under G. Muratori and P. Query for their assistance in the construction of the XDC; E. David for the drawings of the XDC; $\mathrm{H}$. Schuhmann for the drawing of the general installation plan of the experiment; H. Herbert and G. Miller for their help with the installation of the experiment. Finally, we are grateful to the Experimental Area Team of the CERN PS Division under J.D. Simon for their work during the installation and running of the experiment. 


\section{REFERENCES}

[1] R. Armenteros et al. (ASTERIX Collab.), A study of $\bar{p}$ interactions at rest in a $\mathrm{H}_{2}$ gas target, CERN Proposal PSCC/80-101 (1980).

[2] R. Armenteros and B. French, High-Energy Physics, Vol. IV, ed. E.H.S. Burhop (Academic Press, London, 1969), p. 284.

[3] P. Lefèvre, Proc. 4th LEAR Workshop on Physics at LEAR with Low-Energy Antiprotons, Villars-sur-Ollon, 1987, ed. C. Amsler et al. (Harwood Academic Publ., Chur, Switzerland, 1988), p. 19, and references therein.

[4] T.B. Day, G.A. Snow and J. Sucher, Phys. Rev. 118 (1960) 864.

[5] E.G. Auld et al., Phys. Lett. 77B (1978) 454.

[6] U. Gastaldi, Nucl. Instrum. Methods 156 (1978) 257; 157 (1978) 441; 176 (1980) 99; 188 (1981) 459.

[7] J. Jeanjean et al., Nucl. Instrum. Methods 117 (1974) 349.

A. Cordier et al., Nucl. Instrum. Methods 133 (1976) 237.

[8] M.Calvetti et al., Nucl. Instrum. Methods 176 (1980) 255.

[9] H. Kalinowsky and M. Ziegler, private communication.

H. Lenz, Diplomarbeit, Universität Mainz (1982), unpublished.

[10] J.B. Lindsay et al., Nucl. Instrum. Methods 156 (1978) 329.

[11] The OC-DEC On-line Manual, CERN-DD Internal Documentation.

[12] S. Cittolin and B.G. Taylor, Proc. Conf. on Microprocessors in Automation and Communications, University of Kent at Canterbury, 1978 [IEEE Conf. Proc. No. 41 (1979)], p. 309.

[13] L. Bird et al., Nucl. Instrum. Methods 166 (1979) 155.

[14] S. Ahmad et al., Nucl. Instrum. Methods 217 (1983) 169.

[15] S. Cittolin and B. Lofstedt, Proc. Topical Conference on the Application of Micropressors to High-Energy Physics Experiments, Geneva, 1981 (CERN 81-07, Geneva, 1981), p. 91.

[16] J.C. Bizot, Comptes-rendus du Forum sur la micro-informatique en Physique Nucleaire et Physique des Particules, Paris, 1983, ed. G. Fontaine (LPC 83-36, Lab. de Phys. Corpusculaire du Collège de France, Paris, 1983), p. 169.

[17] S. Ahmad et al. (ASTERIX Collab.), Phys. Lett. 157B (1985) 333.

[18] R. Rückl and C. Zupančič, Phys. Lett. 150B (1985) 225.

[19] M. Doser et al. (ASTERIX Collab.), Nucl. Phys. A486 (1988) 493.

[20] U. Schaefer et al. (ASTERIX Collab.), X-rays from ppp Annihilation into Charged Final States, Z. Phys. (1989), in print.

[21] M. Ziegler et al. (ASTERIX Collab.), Phys. Lett. 206B (1988) 151.

[22] G. Reifenröther et al. (ASTERIX Collab.), Phys. Lett. 214B (1988) 325.

[23] M. Doser et al. (ASTERIX Collab.), Phys. Lett. 215B (1988) 792.

[24] B. May et al. (ASTERIX Collab.), $\bar{p}$ p annihilation at rest in $\mathrm{H}_{2}$ gas into $\pi^{+} \pi^{-} \pi^{0}$, submitted to Nucl. Phys. A.

[25] B. May et al. (ASTERIX Collab.), Observation of an isoscalar meson AX(1565) in annihilation of the $\overline{\mathrm{p} p}$ atom from P-states, to be published in Phys. Lett. B (1989).

[26] K.D. Duch et al. (ASTERIX Collab.), Observation and analysis of the E meson in $\bar{p} p$ annihilation at rest in a gaseous hydrogen target at normal pressure and temperature, submitted to Z. Phys. C.

[27] S. Ahmad et al., Phys. Lett. 152B (1985) 135.

[28] U. Gastaldi, Nucl. Phys. A478 (1988) 813.

R. Landua, Proc. of the First Workshop on Antimatter Physics at Low Energy (Fermi Nat. Acc. Lab., Batavia, Illinois, 1986), p. 35. 
Table 1

Characteristics of cylindrical MWPCs

\begin{tabular}{|l|l|l|l|l|l|l|l|}
\cline { 2 - 7 } \multicolumn{1}{c|}{} & \multicolumn{1}{c|}{$\mathrm{C}_{1}$} & $\mathrm{C}_{2}$ & $\mathrm{Q}_{1}$ & \multicolumn{1}{c|}{$\mathrm{Q}_{2}$} & \multicolumn{1}{|c|}{$\mathrm{P}_{1}$} & \multicolumn{1}{c|}{$\mathrm{Q}_{3}$} & \multicolumn{1}{c|}{$\mathrm{P}_{2}$} \\
\hline Useful length (mm) & 740 & 740 & 756 & 1040 & 960 & 1040 & 1070 \\
Anode diameter (mm) & 385.5 & 540.5 & 733.8 & 1189 & 1400 & 1611 & 1742 \\
Cathode gap (mm) & 6 & 6 & 6 & 6 & 6 & 6 & 6 \\
Anode wire spacing (mm) & 2.10 & 2.21 & 2.001 & 1.945 & 2.29 & 2.196 & 2.03 \\
Cathode wire spacing (mm) & 3.45 & 3.13 & 2.9 & 2.8 & - & 3.33 & - \\
Anode wires & 576 & 768 & 1152 & 1920 & 1920 & 2304 & 2688 \\
Cathode wires/plane & 288 & 384 & 576 & 960 & - & 1152 & - \\
Cyl. proj. angle ( $\left.{ }^{\circ}\right)$ & 35 & 45 & 46.73 & 45.92 & - & 49.38 & - \\
& & & & & & & \\
---: No cathode plane & & & & & & & \\
\hline
\end{tabular}

Table 2

Characteristics of each

position-sensitive gamma detector

Anode-cathode gap

$5 \mathrm{~mm}$

Anode wire spacing

$4 \mathrm{~mm}$

Cathode strip width

$4 \mathrm{~mm}$

Cathode-anode angle

$\pm 60^{\circ}$

Number of anode wires/cathode strips

$320 / 320 / 320$

Sensitive area

$1.3 \mathrm{~m}^{2}$ 


\section{Table 3}

Contributions to the readout time of a four-prong event (in $\mu$ s)

$\begin{aligned} & \text { PDP-11/60 interrupt response time } \\ & \text { Readout RMH (DMA transfer) }\end{aligned}$
$\quad 150$ words $\times 2.8 \mu \mathrm{s}$
$\begin{aligned} & \text { Readout XDC (DMA transfer) } \\ & \quad 500 \text { words } \times 2.8 \mu \mathrm{S}\end{aligned}$
$\begin{aligned} & \text { Readout Q-chamber cathodes (DMA transfer) } \\ & \quad 20 \text { words } \times 2.8 \mu \mathrm{S}\end{aligned}$
$\begin{aligned} & \text { Readout GA } 103 \text { (DMA transfer) } \\ & \quad 20 \text { words } \times 2.8 \mu \mathrm{S}\end{aligned}$
$\begin{aligned} & \text { DMA overhead } \\ & \quad \text { Preparation and completion: } 4 \times 1350 \mu \mathrm{S}\end{aligned}$
$\begin{aligned} & \text { Readout scalers, pattern registers } \\ & \quad 30 \text { words } \times 15 \mu \mathrm{s}\end{aligned}$
$\begin{aligned} & \text { DAQ re-enabling time } \\ & \quad \text { Clear buffers, enable new trigger }\end{aligned}$
$\quad 5400$
TOTAL TIME

\section{Table 4}

Summary of performances of the ASTERIX detector

X-rays:

Detection efficiency

Energy resolution (FWHM)

Charged particles:

Solid angle $\left(\mathrm{Q}_{3}\right)$

Momentum resolution

$\mathrm{dE} / \mathrm{dx}$ resolution

Secondary vertex separation

Annihilation vertex resolution

Missing-mass resolution
$25 \%$ at $1.7 \mathrm{keV} ; 50 \%$ at $5.5 \mathrm{keV} ; 15 \%$ at $10 \mathrm{keV}$ $45 \%$ at $1.7 \mathrm{keV} ; 25 \%$ at $5.5 \mathrm{keV} ; 20 \%$ at $10 \mathrm{keV}$

$50 \% \times 4 \pi$

$\sigma=4.2 \%$ at $1 \mathrm{GeV} / \mathrm{c}$

$\sigma=14 \%$ (for minimum-ionizing particles)

$\sigma_{\mathrm{xy}}=2.4 \mathrm{~mm} ; \sigma_{\mathrm{z}}=7 \mathrm{~mm}$

$\sigma_{\mathrm{xy}}=0.7 \mathrm{~mm} ; \sigma_{\mathrm{z}}=2.1 \mathrm{~mm}$ (four-prong events)

$\pi^{+} \pi^{-} \pi^{0}: \sigma=0.06 \mathrm{GeV}^{2}$

$\pi^{+} \pi^{-} \pi^{+} \pi^{-} \pi^{0}: \sigma=0.0012 \mathrm{GeV}^{2}$

$\gamma$-rays:

Solid angle

Detection efficiency
$75 \% \times 4 \pi$

$25 \%$ (above $150 \mathrm{MeV}$ ) 
Table 5

ASTERIX data-taking at LEAR

\begin{tabular}{|c|c|c|c|c|c|c|}
\hline $\begin{array}{l}\text { Run } \\
\text { period }\end{array}$ & $\begin{array}{c}\overline{\mathrm{p}} \text { beam } \\
\text { momentum } \\
(\mathrm{MeV} / \mathrm{c})\end{array}$ & $\begin{array}{l}\text { Number of } \\
\text { spills }^{\text {a) }}\end{array}$ & $\begin{array}{l}\text { Target } \\
\text { gas }\end{array}$ & Main trigger ${ }^{b)}$ & $\begin{array}{c}\text { Raw events } \\
\text { on tape } \\
\left(\times 10^{6}\right)\end{array}$ & Comments \\
\hline Dec. 83 & $\begin{array}{l}609 \\
309\end{array}$ & $\begin{array}{l}19 \\
52\end{array}$ & $\begin{array}{l}\mathrm{H}_{2} \\
\mathrm{H}_{2}\end{array}$ & $\begin{array}{l}\overline{\mathbf{p}} \text { Stop } \\
\text { Mult(=0) } \times 1 \text { gap } \\
\overline{\mathrm{p}} \text { Stop } \\
\overline{\mathrm{p}} \text { Stop } \times 1 \text { gap }\end{array}$ & $\begin{array}{l}0.5 \\
0.1 \\
3.5\end{array}$ & $\begin{array}{l}\text { Debugging } \\
\text { K X-rays }\end{array}$ \\
\hline Mar. 84 & 309 & 127 & $\mathrm{H}_{2}$ & $\begin{array}{l}\overline{\mathrm{p}} \text { Stop } \times \text { ACC veto } \\
\text { Mult }(\geq 3) \times \text { ACC } \\
\text { veto }\end{array}$ & $\begin{array}{l}3.8 \\
8.8\end{array}$ & \\
\hline Aug. 84 & 200 & 101 & $\begin{array}{l}\mathrm{H}_{2} \\
\\
\mathrm{~N}_{2}\end{array}$ & $\begin{array}{l}\overline{\mathrm{p}} \text { Stop } \\
\text { Mult }(\geq 2) \\
\text { Mult }(\geq 2) \times 1 \text { gap } \\
\text { Mult }(\geq 5) \\
\overline{\mathbf{p}} \text { Stop }\end{array}$ & $\begin{array}{l}3.0 \\
3.6 \\
3.0 \\
1.2 \\
0.7\end{array}$ & \\
\hline Oct. 85 & 105 & 193 & $\mathrm{D}_{2}$ & $\begin{array}{l}\text { Mult }(=2) \\
\text { Mult }(=2) \times 1 \text { gap } \\
\text { Mult }(=2) \times \text { GAl03 } \\
(2 \gamma) \\
\text { Mult }(=4) \\
\text { Mult }(=4) \times 1 \text { gap } \\
\text { Mult }=0) \times 1 \text { gap } \\
\text { Mult }(=2) \\
\text { Mult(=4) }\end{array}$ & $\begin{array}{l}2.4 \\
2.5 \\
0.7 \\
\\
1.8 \\
8.0 \\
0.4 \\
0.5 \\
0.6\end{array}$ & $\bar{p} \mathrm{~d} X$-rays \\
\hline June 86 & 105 & 156 & $\mathrm{H}_{2}$ & $\begin{array}{l}\text { Mult }(=0) \times 1 \text { gap } \\
\text { Mult }(=2) \times \\
\text { GA103 }(\eta \rightarrow \gamma \gamma) \\
\text { Mult }(=4) \times 1 \text { gap } \\
\text { Mult }(=4) \times \\
\text { GA103(4 } 4 \eta+\pi \pi \mathrm{KK}) \\
\text { Mult }(=6)\end{array}$ & $\begin{array}{l}3.2 \\
1.9 \\
3.2 \\
1.0 \\
0.8\end{array}$ & $\begin{array}{l}\mathrm{L}+\mathrm{K}_{\alpha} \\
\mathrm{X} \text {-rays }\end{array}$ \\
\hline TOTAL: & & 648 spills & & & $\begin{array}{l}55.2 \text { million events } \\
\text { (830 tapes) }\end{array}$ & \\
\hline
\end{tabular}

a) 'Spill': Accounting unit $=$ extraction of stored $\bar{p}$ 's within $45-60 \mathrm{~min}$; intensity (ASTERIX) $\approx 10^{4}-10^{5} \mathrm{~s}^{-1}$.

b) Trigger code abbreviations:
$\overrightarrow{\mathrm{p}}$ Stop:
$\operatorname{Mult}(=\mathrm{n})$ :
Minimum-bias trigger (in 1985 and 1986, every eighth event on tape was $\bar{p}$ Stop).
1 gap:
Charged-particle multiplicity $n$ requested in MWPCs $\mathrm{C}_{1}, \mathrm{C}_{2}, \mathrm{Q}_{2}$, and $\mathrm{P}_{1}$.
$\mathrm{X}$-ray candidate (isolated hit on one XDC wire, no hit on neighbouring wires).
ACC veto:
Veto of ACC 2160 processor on heavily ionizing particles in XDC (scattered $\overline{\mathrm{p}}$ 's).
GA103: Intelligent trigger on final state (e.g. $2 \gamma$ 's, missing mass of $\eta$ ). 


\section{Figure captions}

Fig. 1 Side and front view of the ASTERIX detector.

Fig. $2 \mathrm{H}_{2}$ target, $\mathrm{XDC}$, and $\mathrm{T}_{1}, \mathrm{~T}_{2}, \mathrm{~T}_{3}$ and $\mathrm{T}_{4}$ scintillators.

Fig. 3 Assembly of the $T_{2}$ and $T_{3}$ scintillators and light-guides.

Fig. 4 Annihilation vertex distribution for $105 \mathrm{MeV} / \mathrm{c}$ antiprotons stopping in the $\mathrm{H}_{2}$ gas target at NTP.

Fig. 5 Schematic view of the XDC across the target axis.

Fig. 6 Event with four charged particles and an X-ray in the XDC. The pulse height of each $32 \mathrm{~ns}$ sample is indicated by the vertical amplitude; the base line of each sample is positioned at the corresponding drift cell and drift-time.

Fig. 7 Position sensitive gamma-ray end-cap detectors.

Fig. 8 Schematic diagram of the trigger.

Fig. 9 Data-acquisition system.

Fig. 10 REMUS readout for the XDC.

Fig. 11 RMH readout for the cylindrical MWPCs.

Fig. $12 \mathrm{Q}$ chamber cathode readout.

Fig. $13 \mathrm{X}$-ray calibration lines from a) ${ }^{54} \mathrm{Mn}$ (5.5 keV and $2.5 \mathrm{keV}$ escape peak); b) ${ }^{57} \mathrm{Co}$ (6.5 keV, $14.4 \mathrm{keV}$, and $3.5 \mathrm{keV}$ escape peak); c) $\mathrm{L}_{\alpha}$-line from protonium singled out in coincidence with M X-ray.

Fig. 14 Drift lines of electrons in the XDC with a magnetic field of $0.8 \mathrm{~T}$. The crosses mark the isochrones for charge collection.

Fig. $15 \mathrm{X}$-ray detection efficiency (for two charged tracks in the final state and $105 \mathrm{MeV} / \mathrm{c} \overline{\mathrm{p}}$ Stop distribution).

Fig. $16 \mathrm{X}$-ray energy spectrum for events with two charged tracks in the final states (the solid line shows the calculated background from inner bremsstrahlung).

Fig. 17 Event display showing reconstructed particle tracks, XDC information, and two gamma rays detected in $\mathrm{Q}_{3} / \mathrm{P}_{\mathbf{2}}$ and the downstream end-cap detector.

Fig. 18 Momentum resolution of the spectrometer for charged particles from Monte Carlo simulations and real collinear events $\overline{\mathrm{p}} \mathrm{p} \rightarrow \pi^{+} \pi^{-}$.

Fig. 19 Direction resolution for charged tracks. The plot shows the measured angle between the two tracks from the reaction $\bar{p} \mathrm{p} \rightarrow \pi^{+} \pi^{-}$.

Fig. $20 \mathrm{dE} / \mathrm{dx}$ distribution for events $\overline{\mathrm{p}} \mathrm{p} \rightarrow \pi^{+} \pi^{-} \mathrm{K}^{+} \mathrm{K}^{-}$.

Fig. 21 Residuals of individual tracks with respect to the annihilation vertex in the $x-y$ plane.

Fig. 22 Residuals of individual tracks with respect to the annihilation vertex in the $\mathrm{z}$ direction.

Fig. 23 Distance of two-prong secondary vertices with respect to the annihilation vertex in the $x-y$ plane.

Fig. 24 Gamma detection efficiency as a function of energy, measured with $\pi^{+} \pi^{-} \pi^{+} \pi^{-} \pi^{0}$ events.

Fig. 25 a) Single-track momentum distribution of collinear events $\bar{p} p \rightarrow \pi^{+} \pi^{-}(928 \mathrm{MeV} / \mathrm{c})$ and $\overline{\mathrm{p} p} \rightarrow \mathrm{K}^{+} \mathrm{K}^{-}(798 \mathrm{MeV} / \mathrm{c})$.

b) Momentum distribution after both tracks were fitted with a single circle (a smaller data sample).

Fig. 26 a) Missing-mass squared recoiling against two charged pions.

b) $2 \gamma$ invariant mass distribution for two-prong events with two reconstructed gammas.

Fig. 27 Missing-mass squared recoiling against four charged pions.

Fig. 28 a) Missing energy of events with four charged particles.

b) Missing momentum of events with four charged particles. The peak at zero missing momentum and energy is from events with four charged pions and no missing particle. 
Fig. $29 \mathrm{~K}^{+} \mathrm{K}^{-}$invariant mass spectrum for $\overline{\mathrm{p} p} \rightarrow \pi^{+} \pi^{-} \mathrm{K}^{+} \mathrm{K}^{-}$in the region of the $\Phi$ meson.

Fig. 30 Invariant mass squared $\pi^{+} \pi^{-} \pi^{0}$ (four entries per event) for $\bar{p} p \rightarrow \pi^{+} \pi^{-} \pi^{+} \pi^{-} \pi^{0}$, requiring a kinematic fit probability $\geq 0.01$. The inset shows the $\eta$ region $\left(0.28-0.34 \mathrm{GeV}^{2}\right)$ enlarged for a bigger data sample.

Fig. 31 Mass resolution of two-pion resonances from the reaction $\bar{p} p \rightarrow \pi^{0} \mathrm{X}$, with $\mathrm{X} \rightarrow \pi^{+} \pi^{-}$ (obtained by Monte Carlo simulations). 


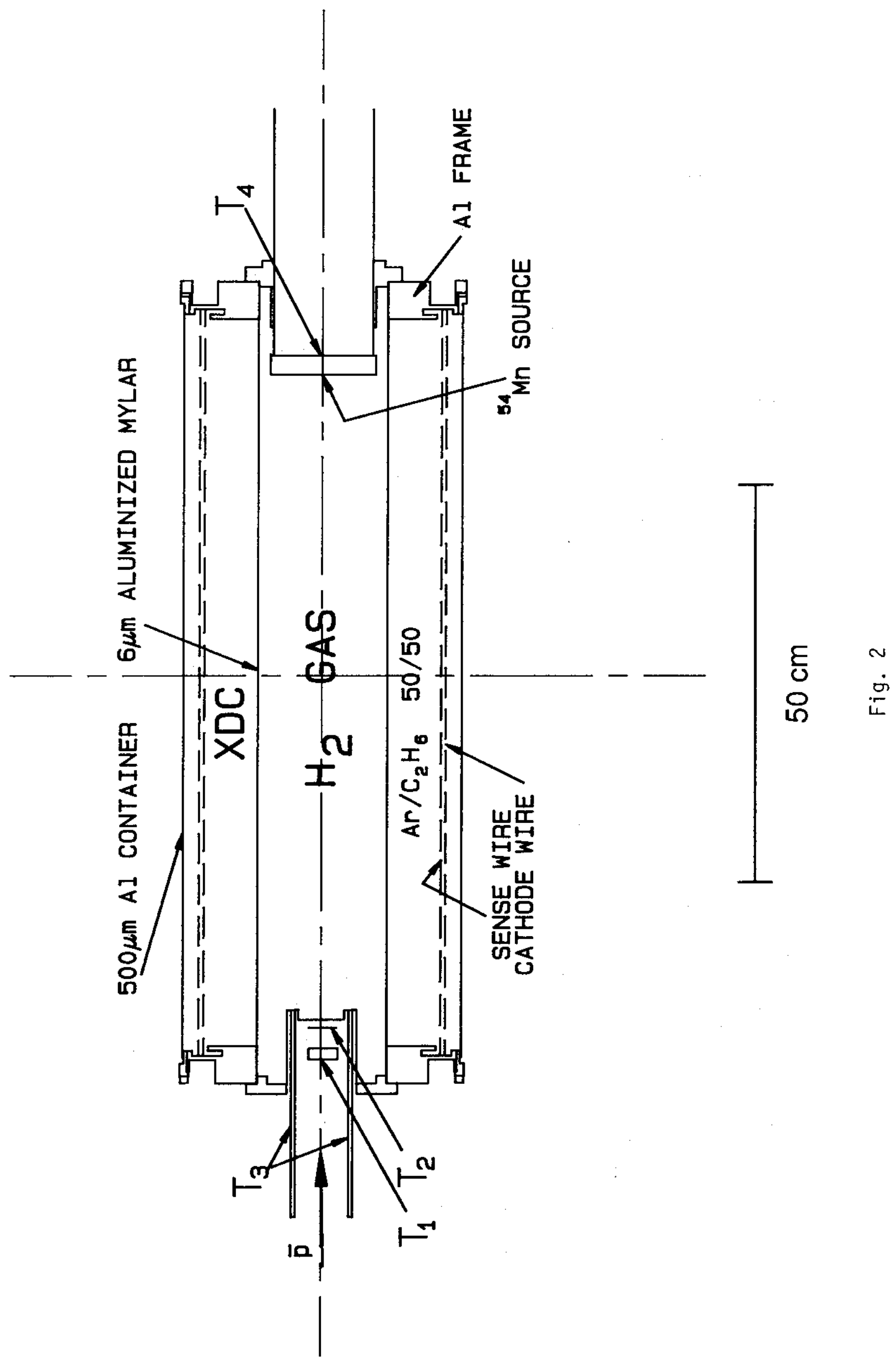




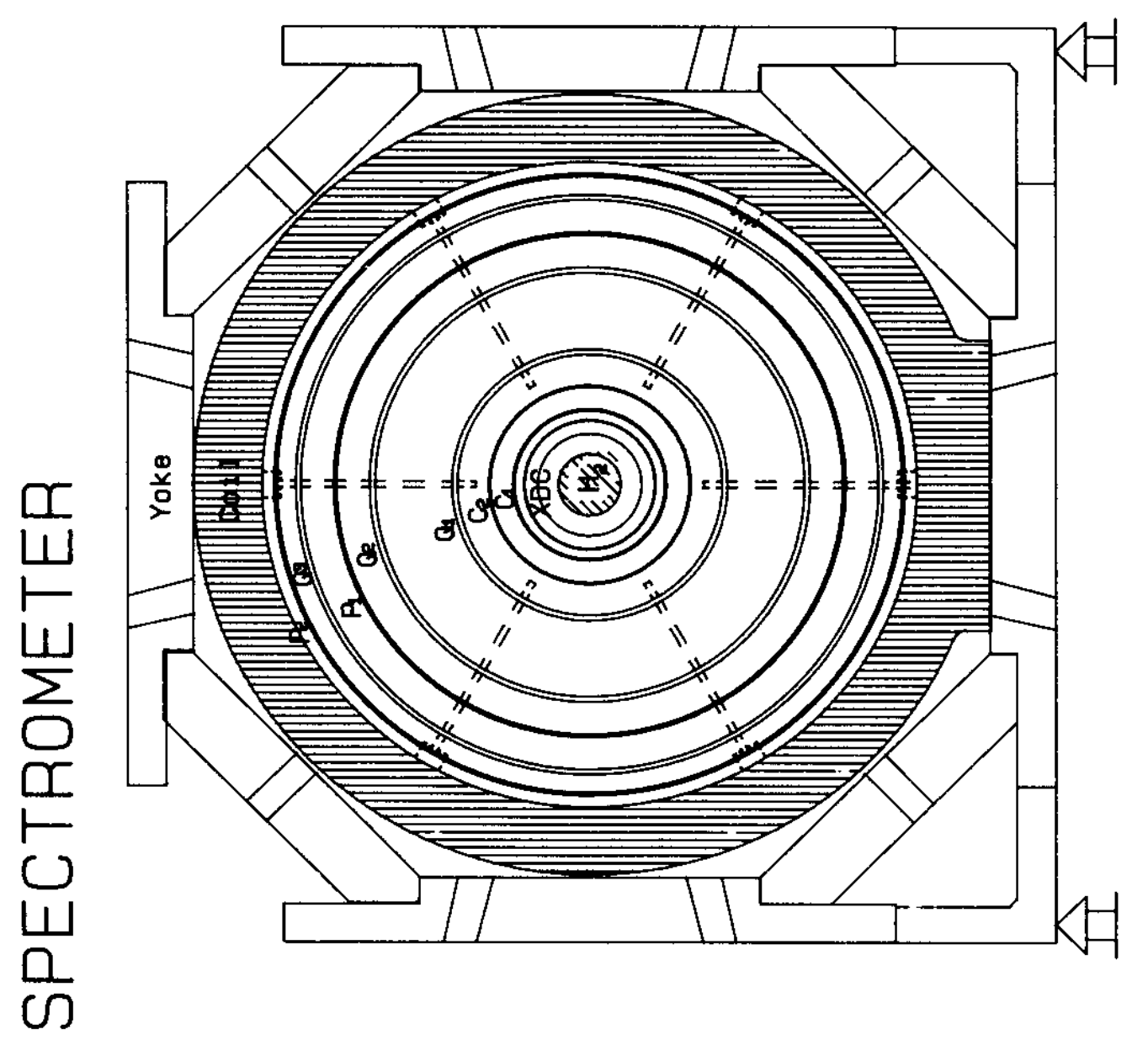

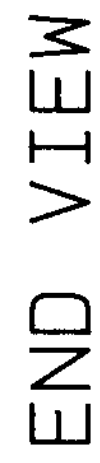
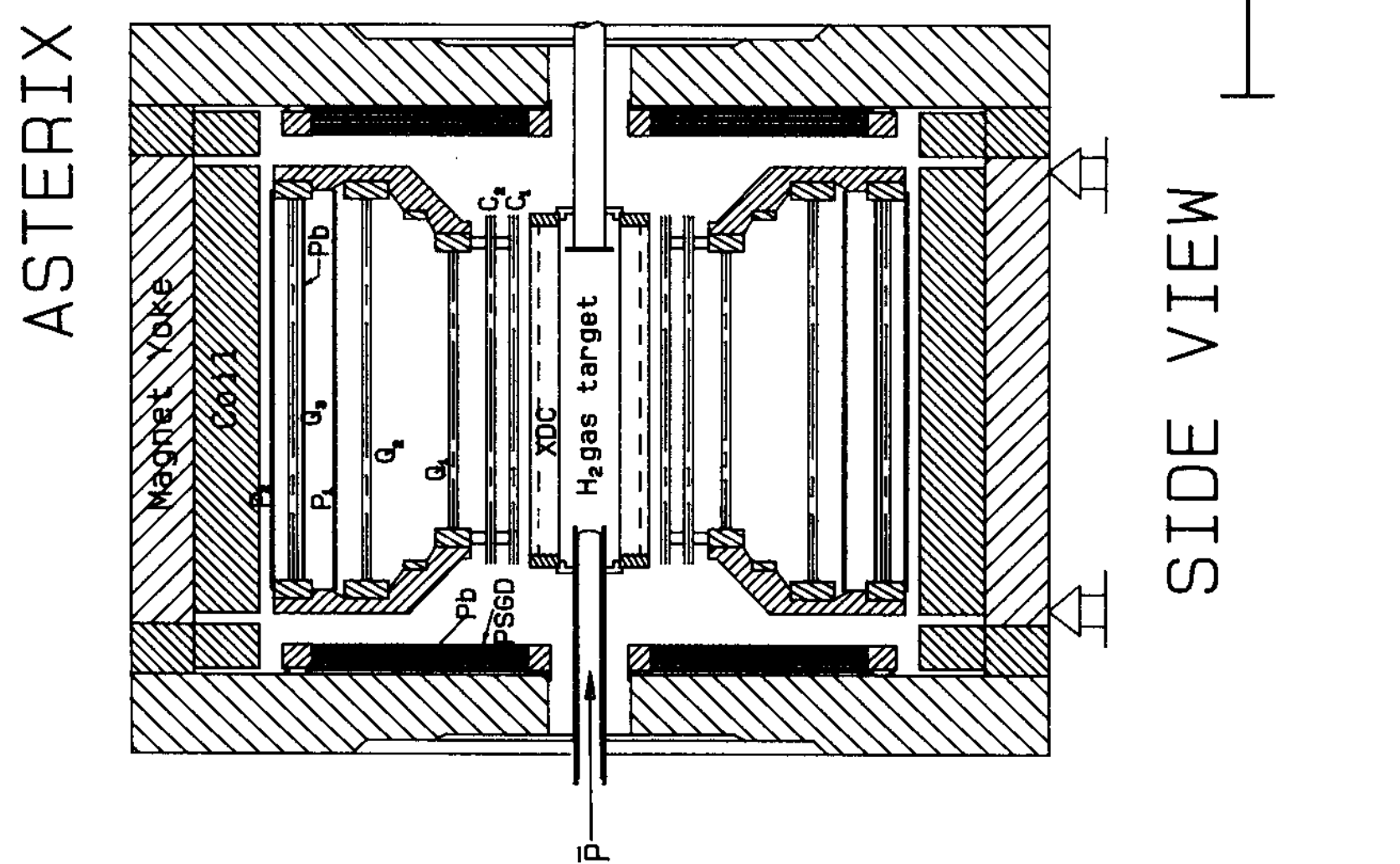

$\varepsilon-$ 


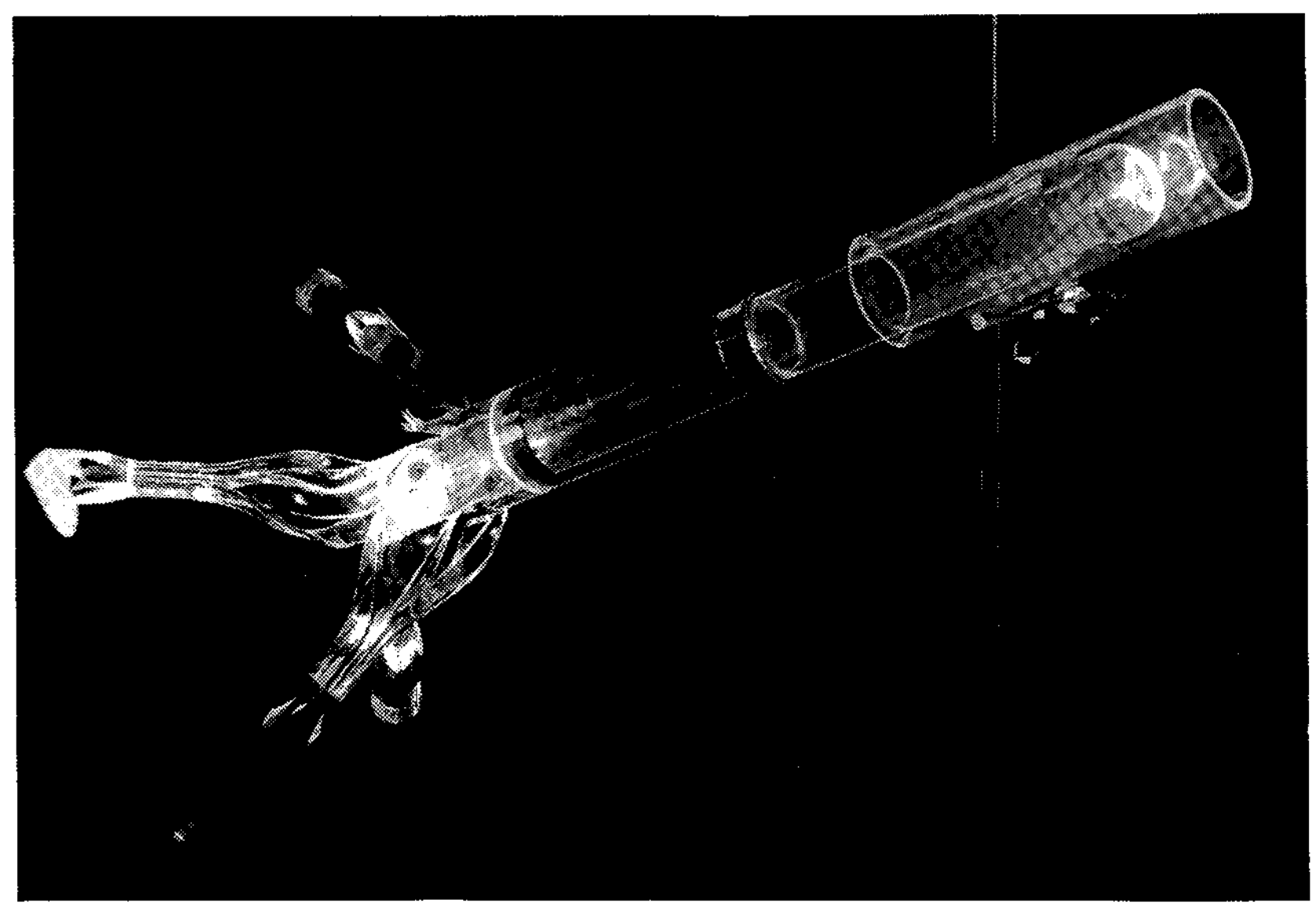

Fig. 3

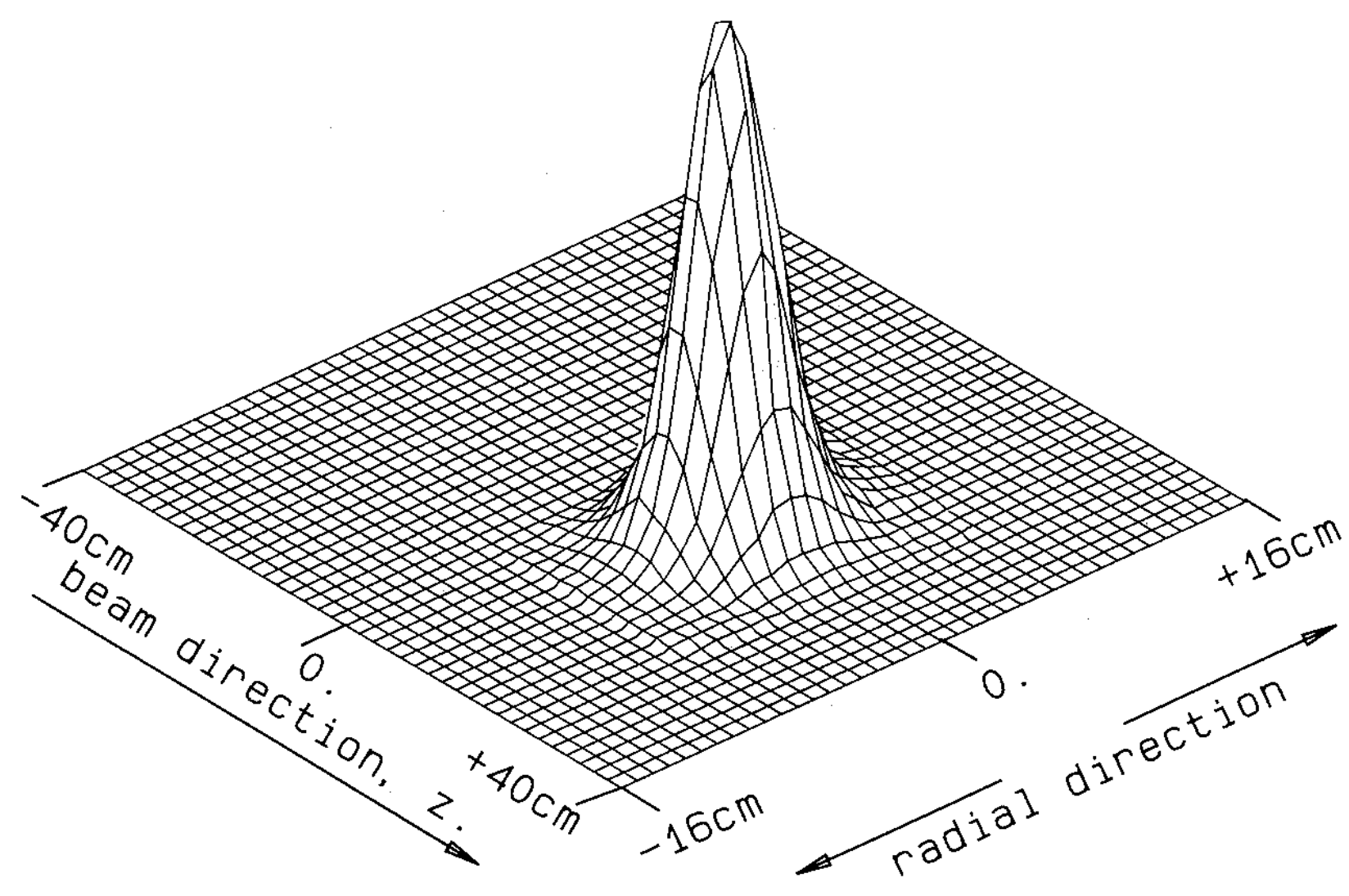

Fig. 4 


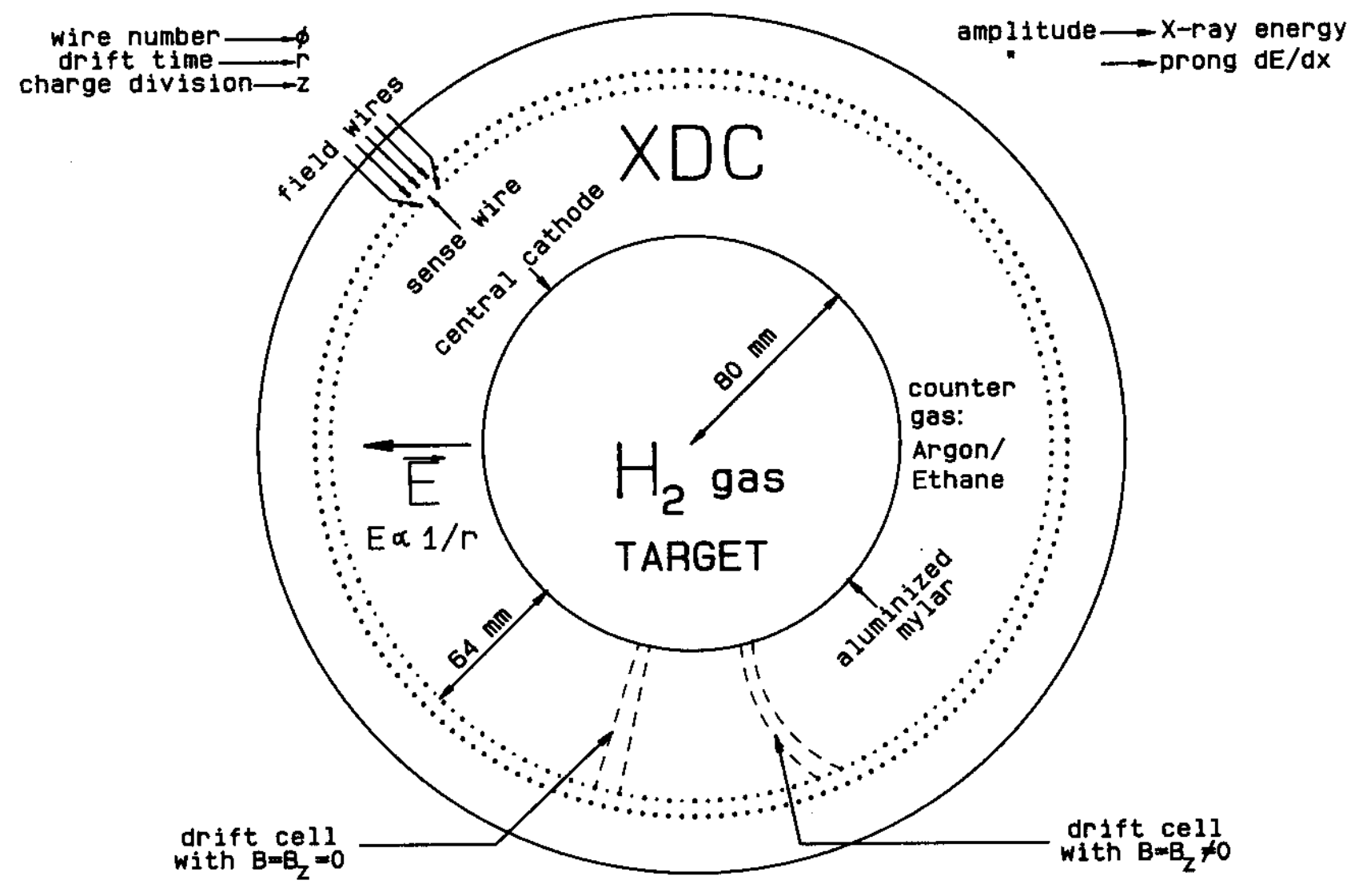

Fig. 5

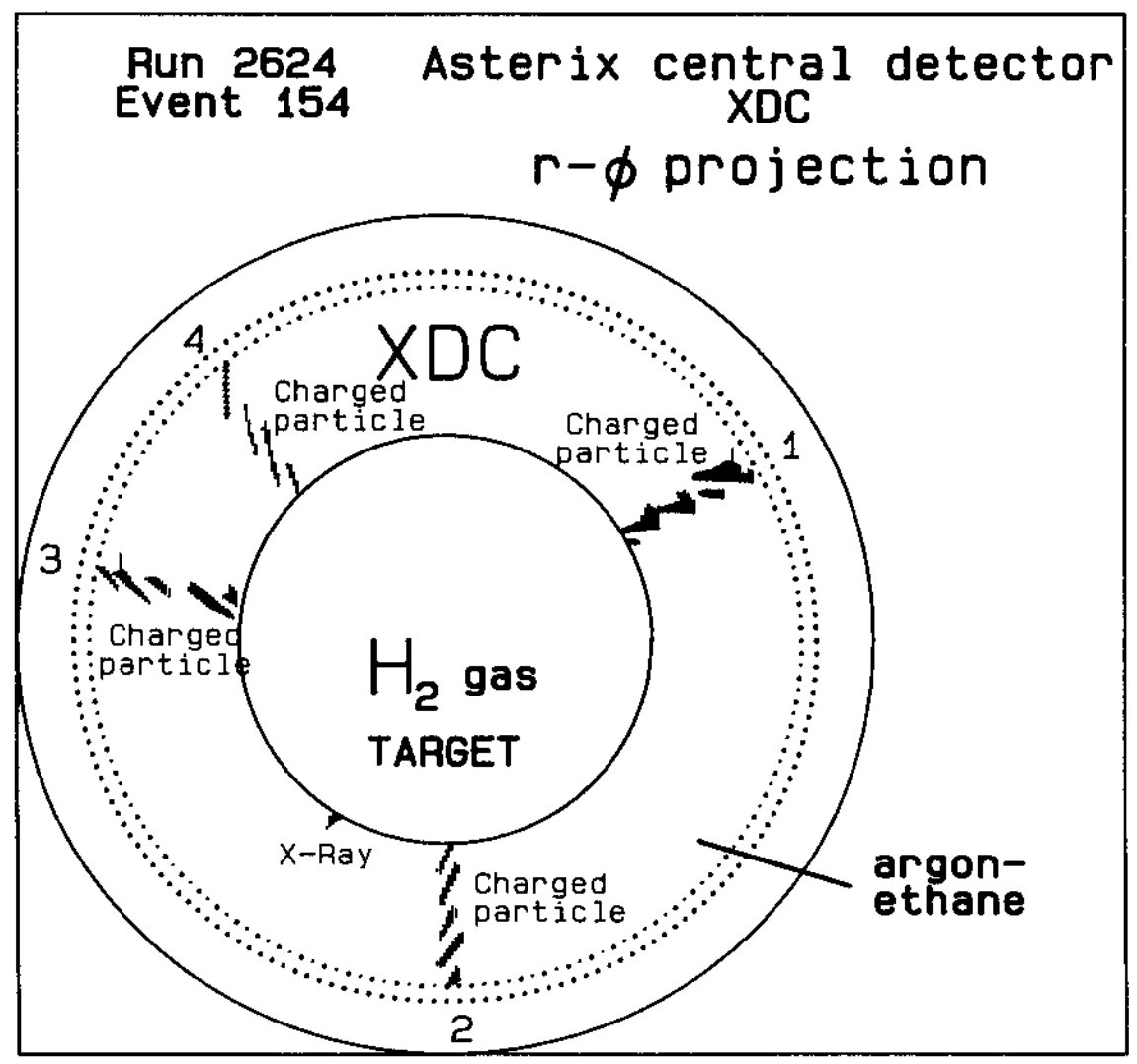

Fig, 6 


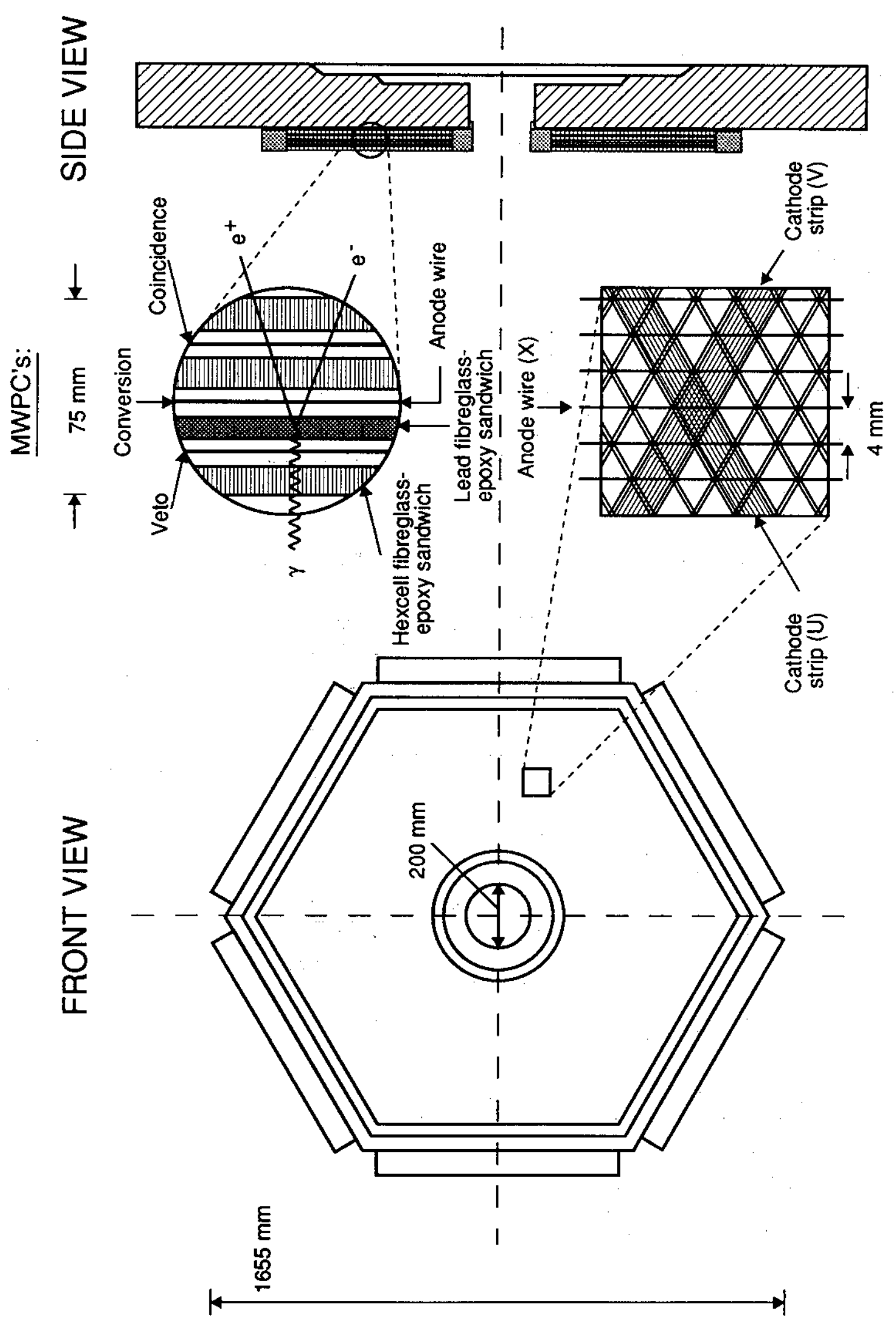

ir 


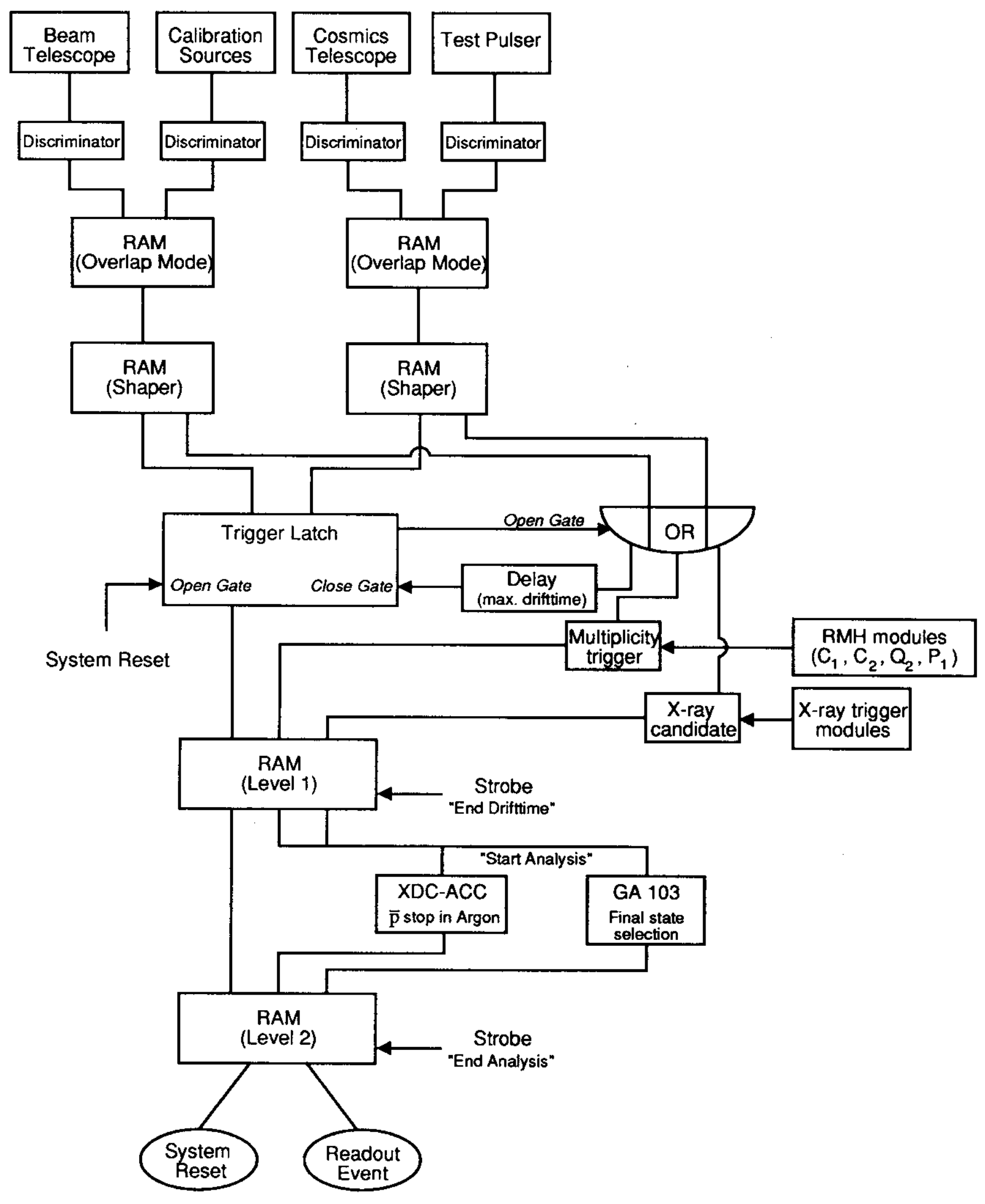

Fig. 8 


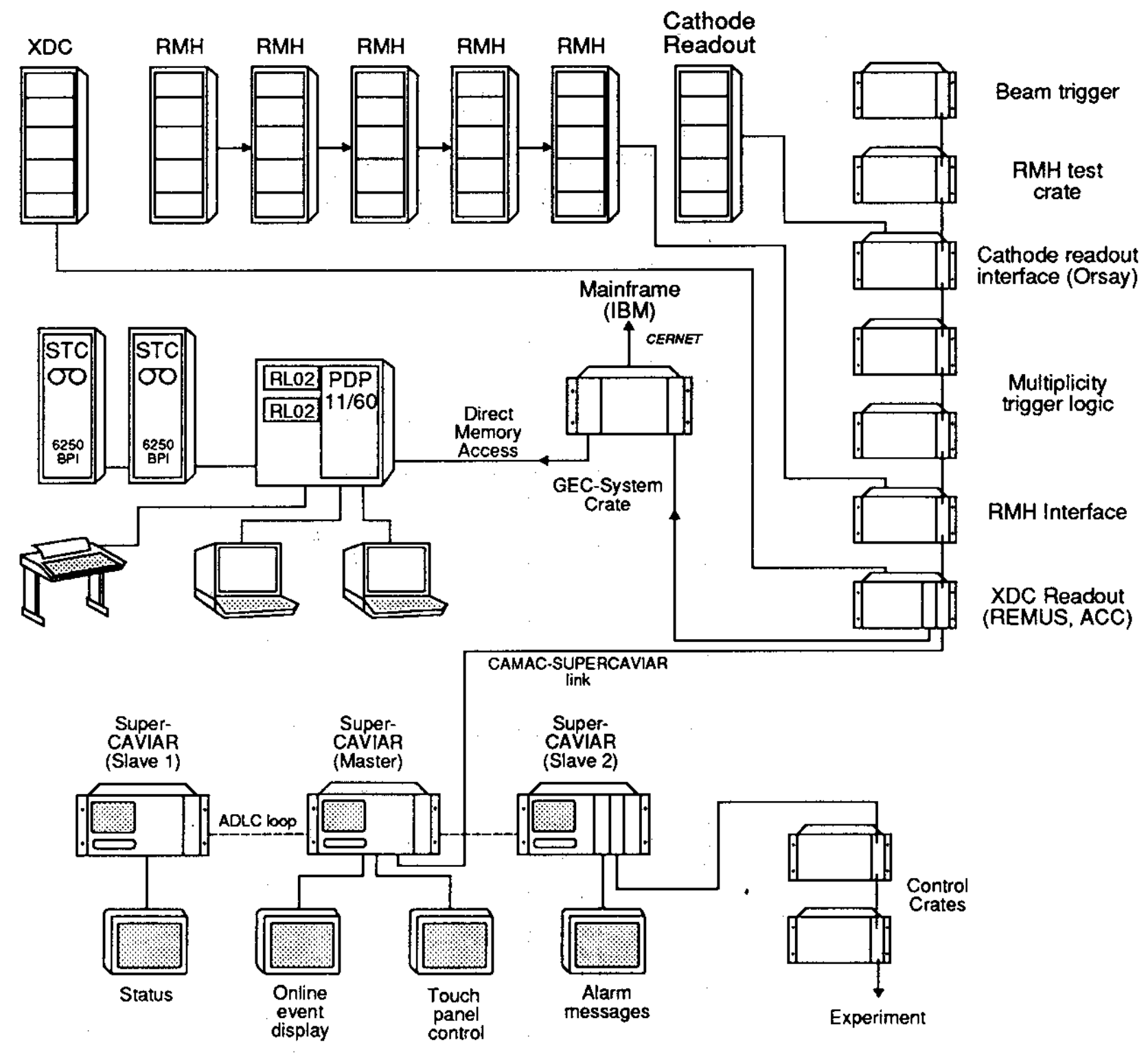

Fig. 9 


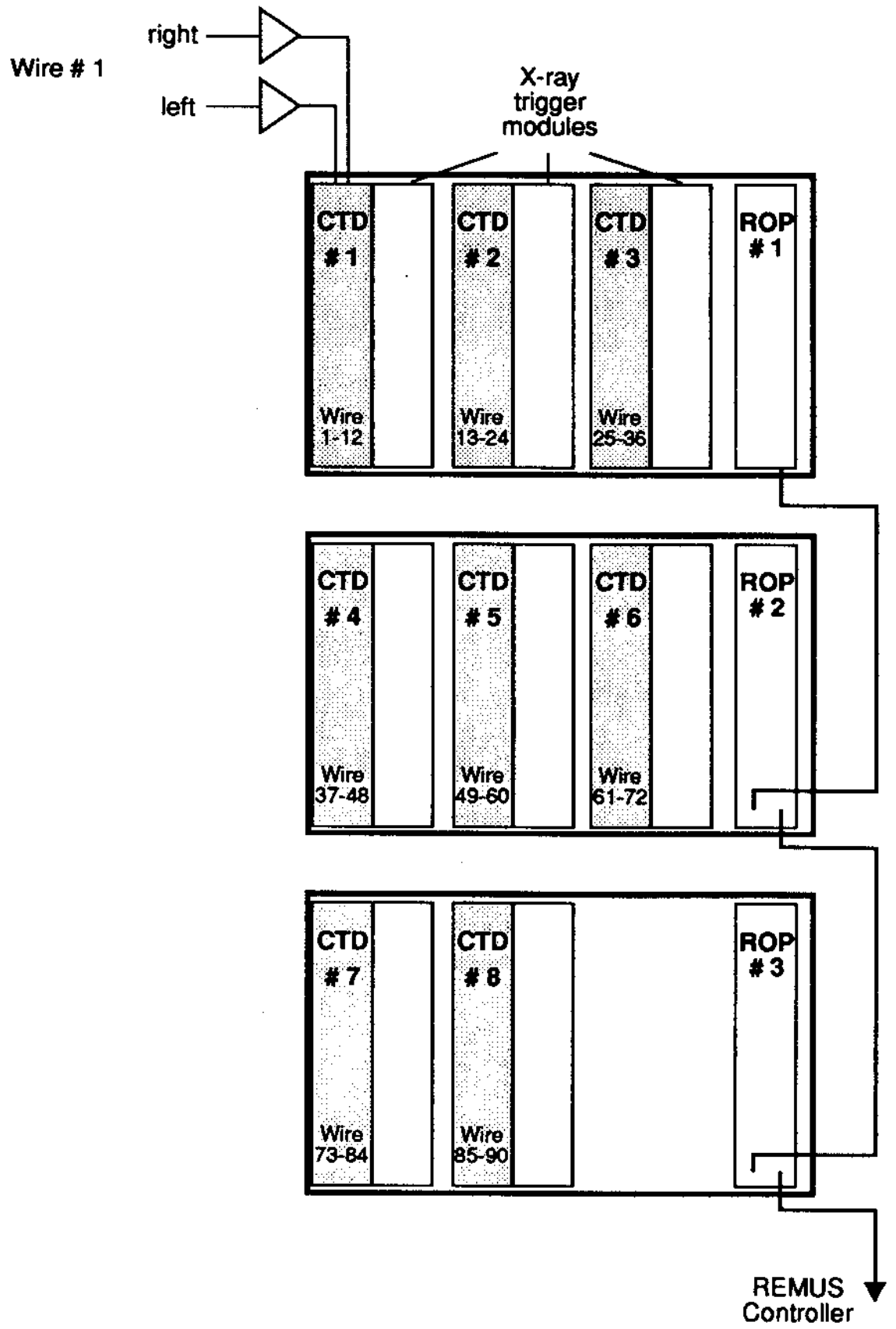

Fig. 10 


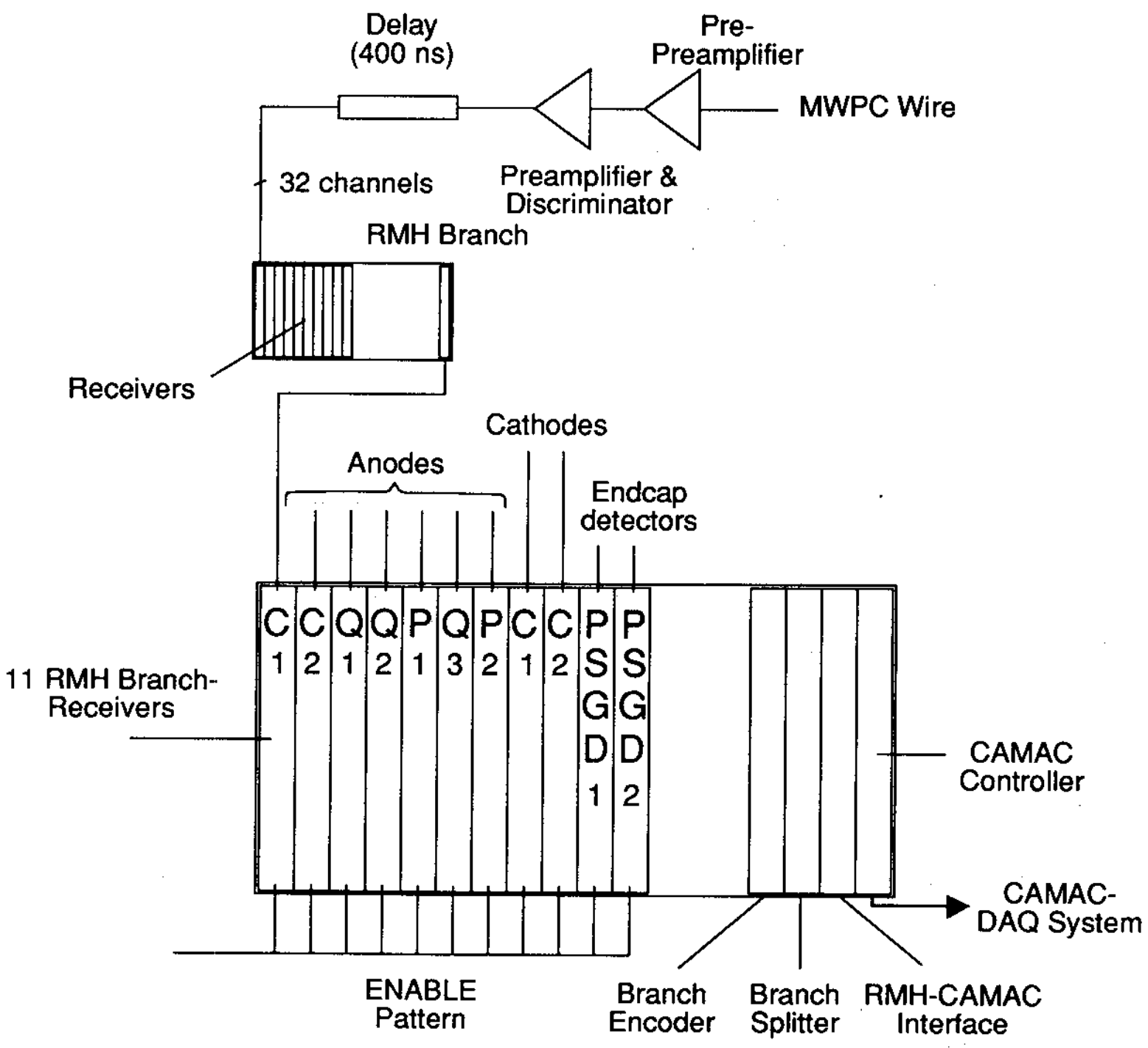

Fig. 11 


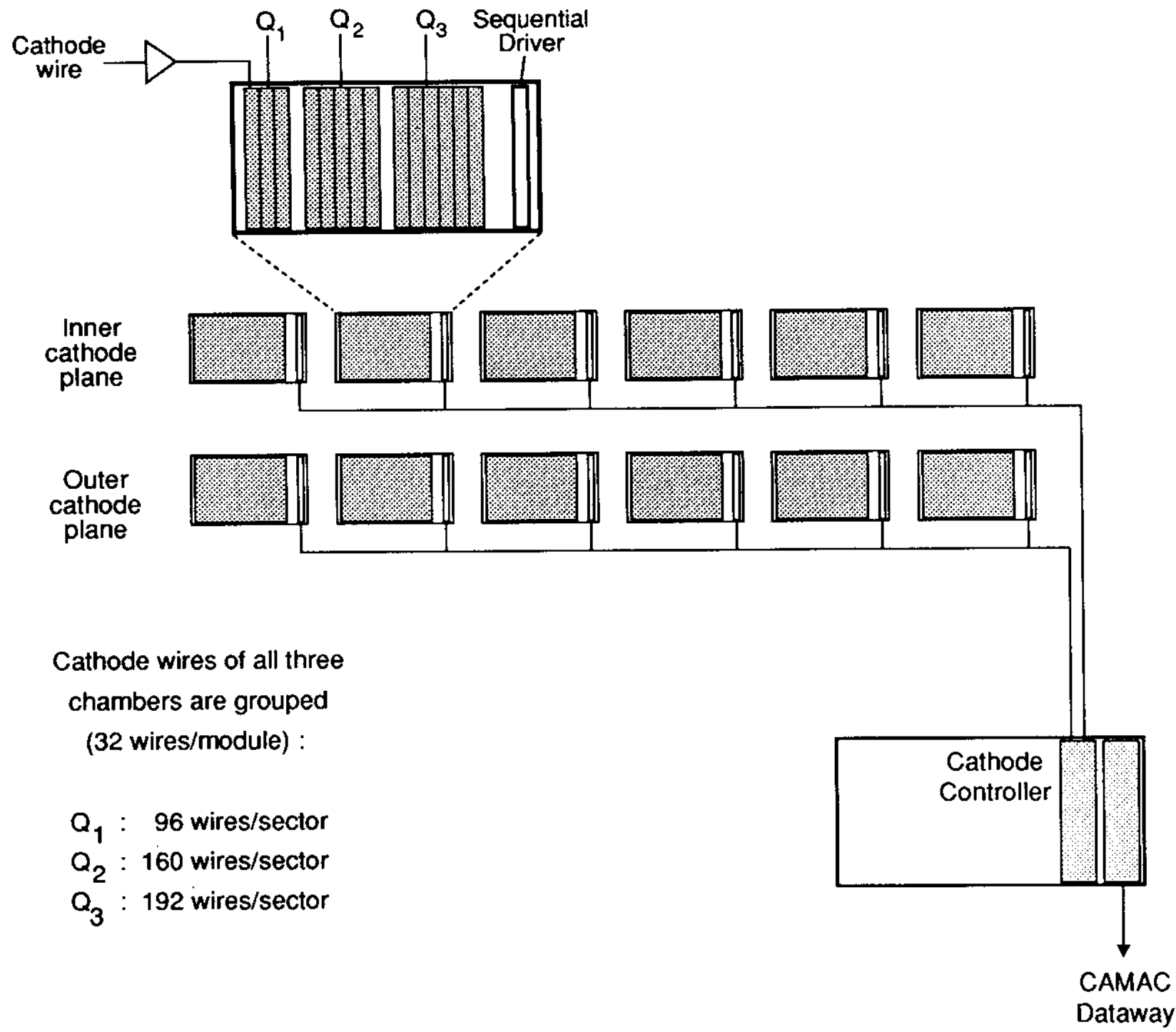

Fig. 12 


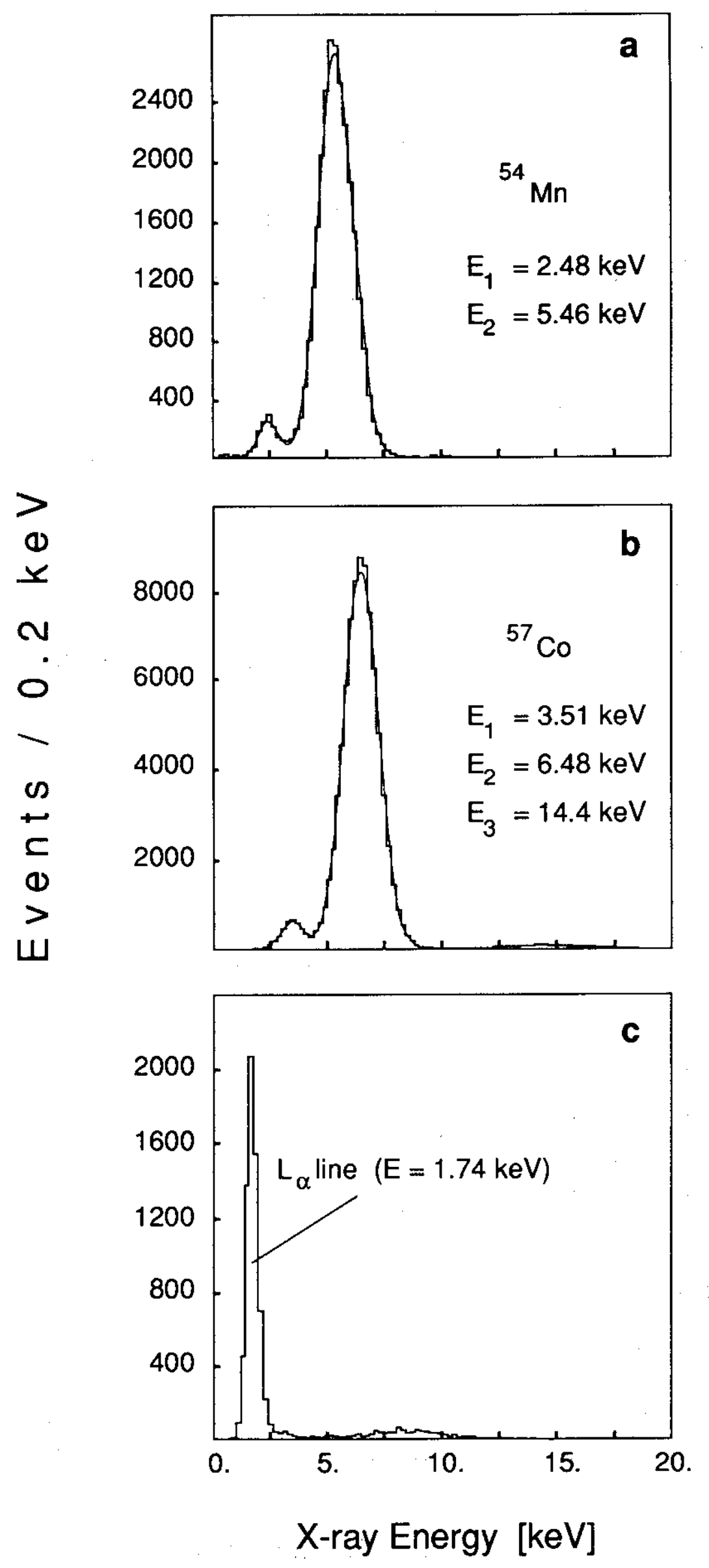

Fig. 13 


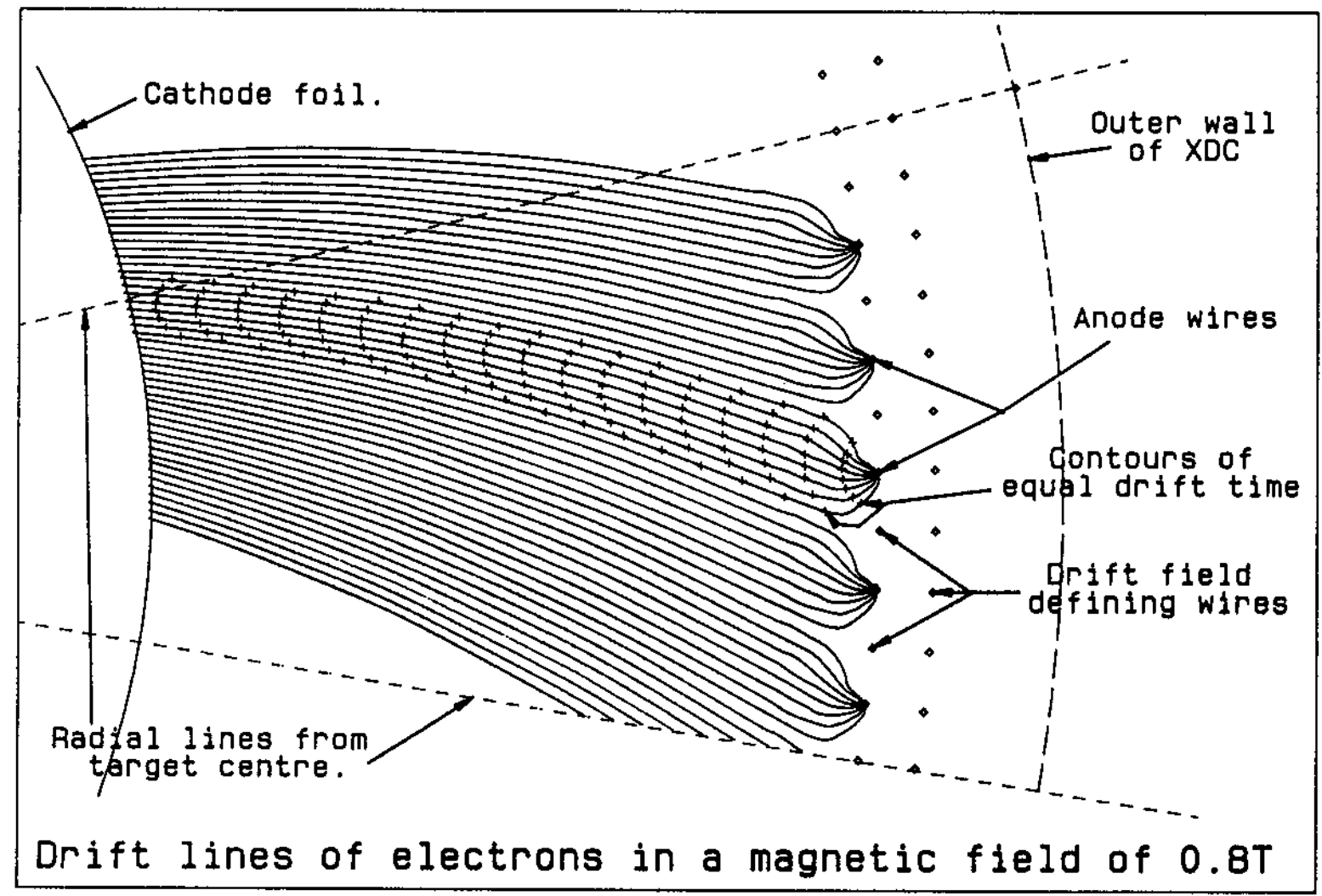

Fig. 14

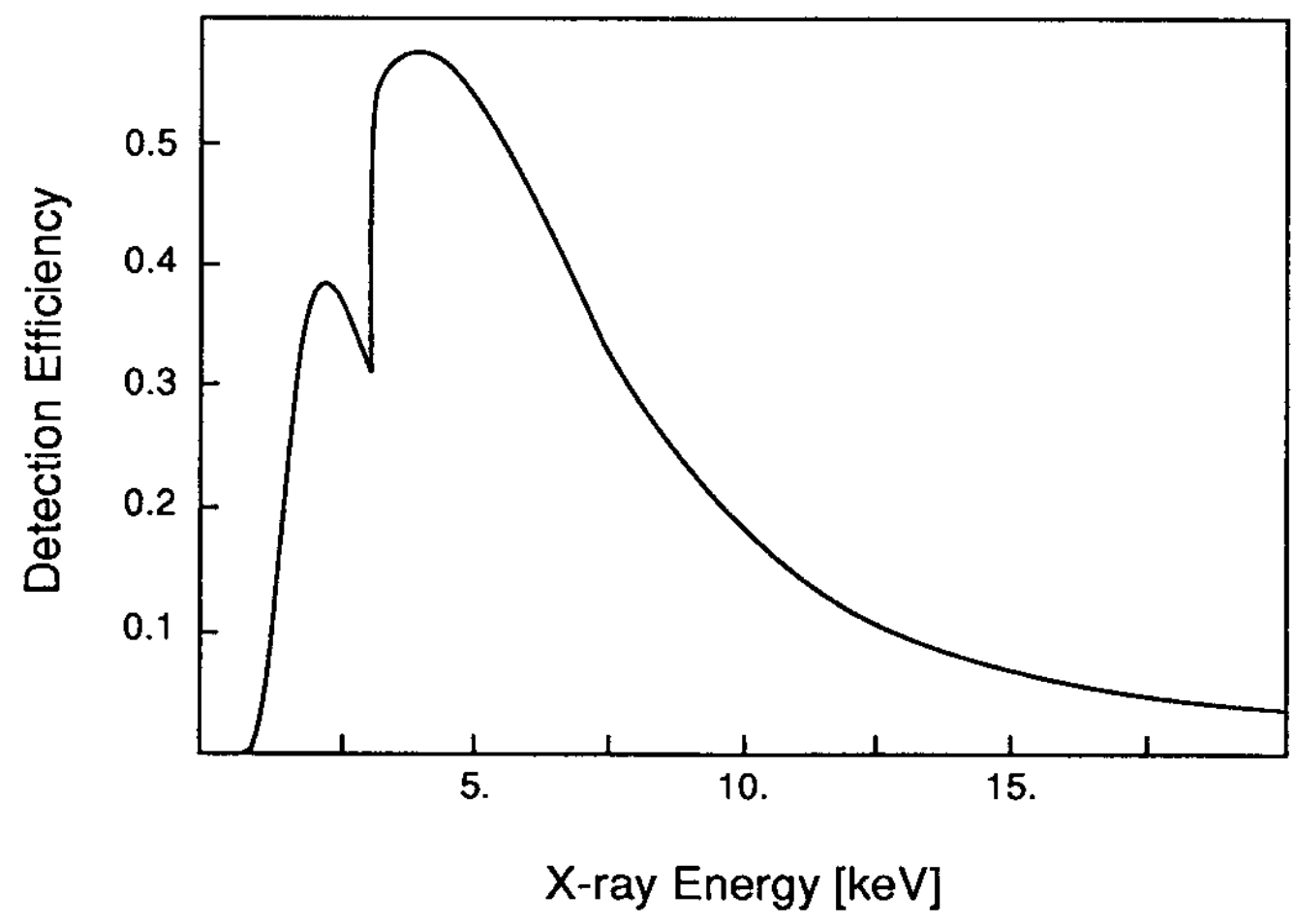

Fig. 15 


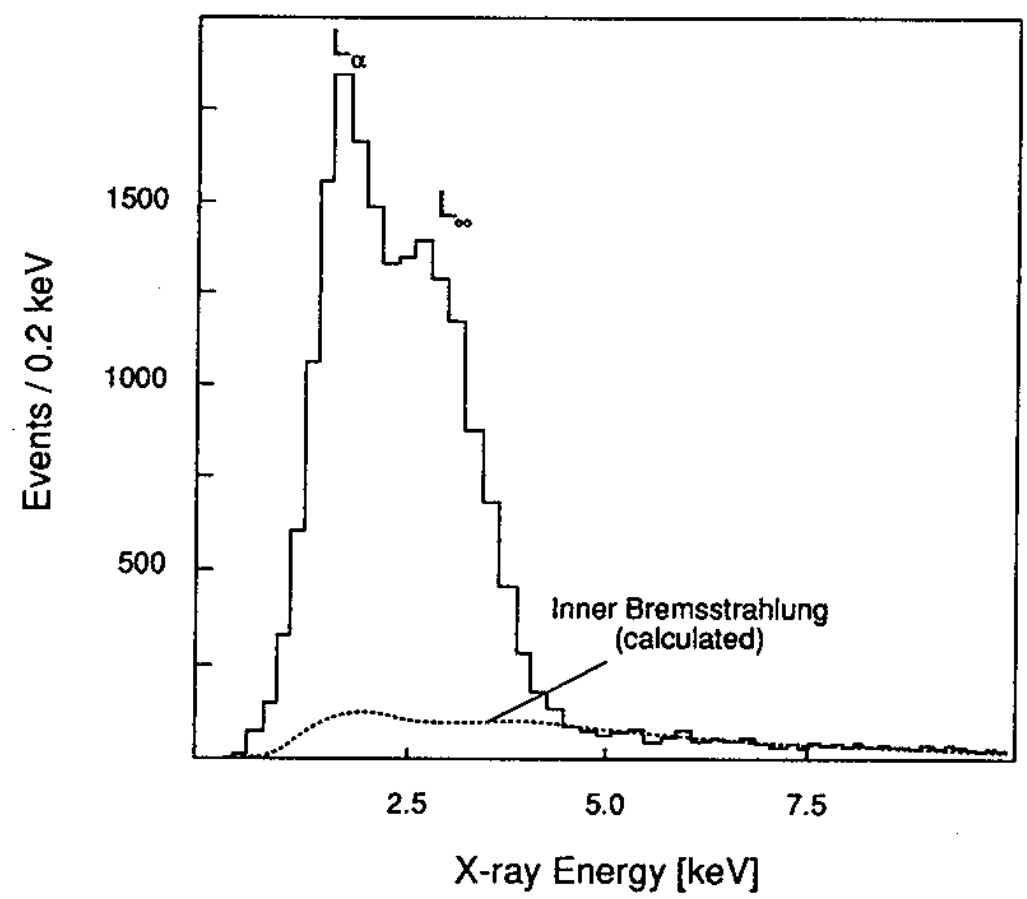

Fig, 16

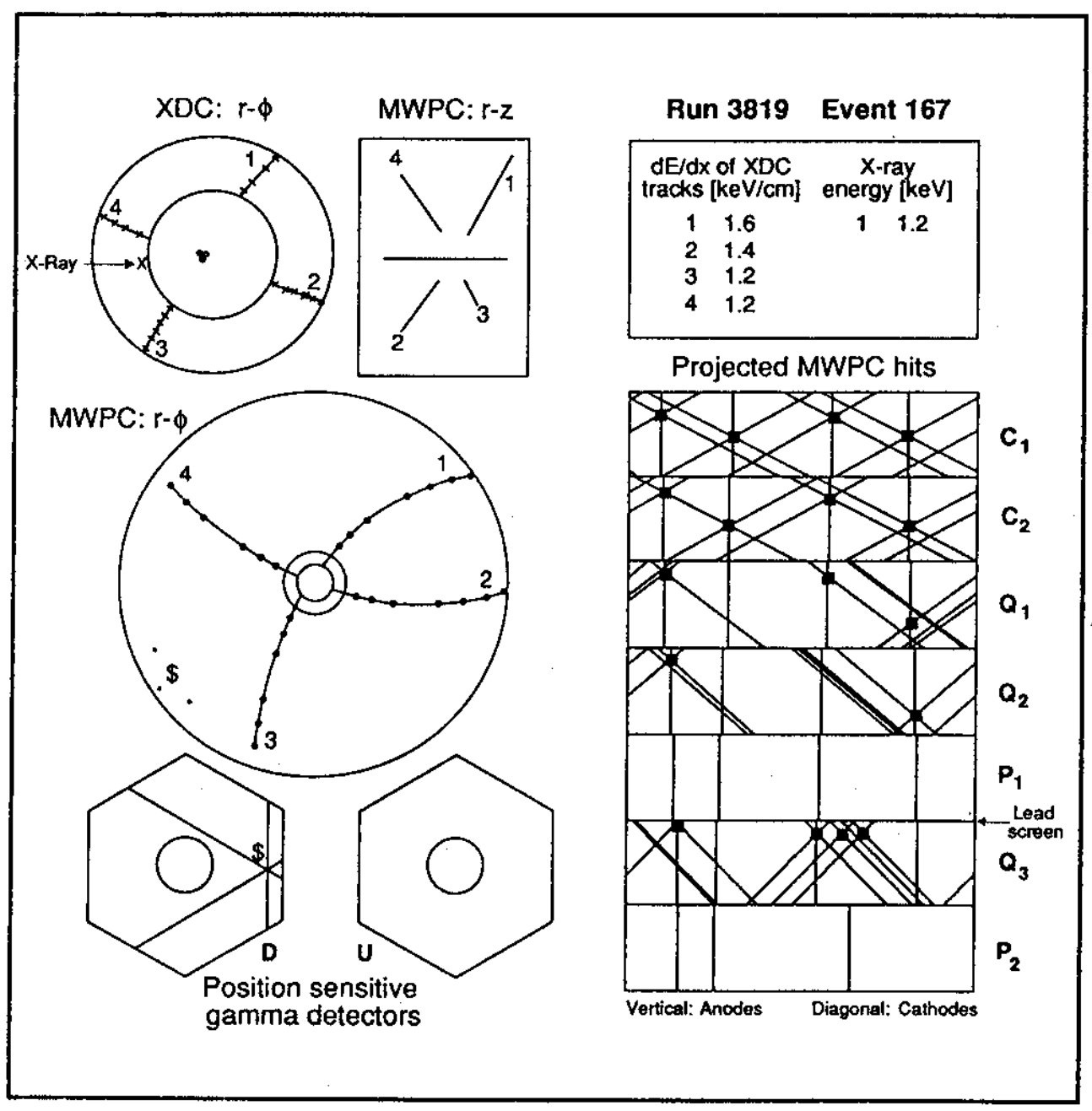

Fig. 17 


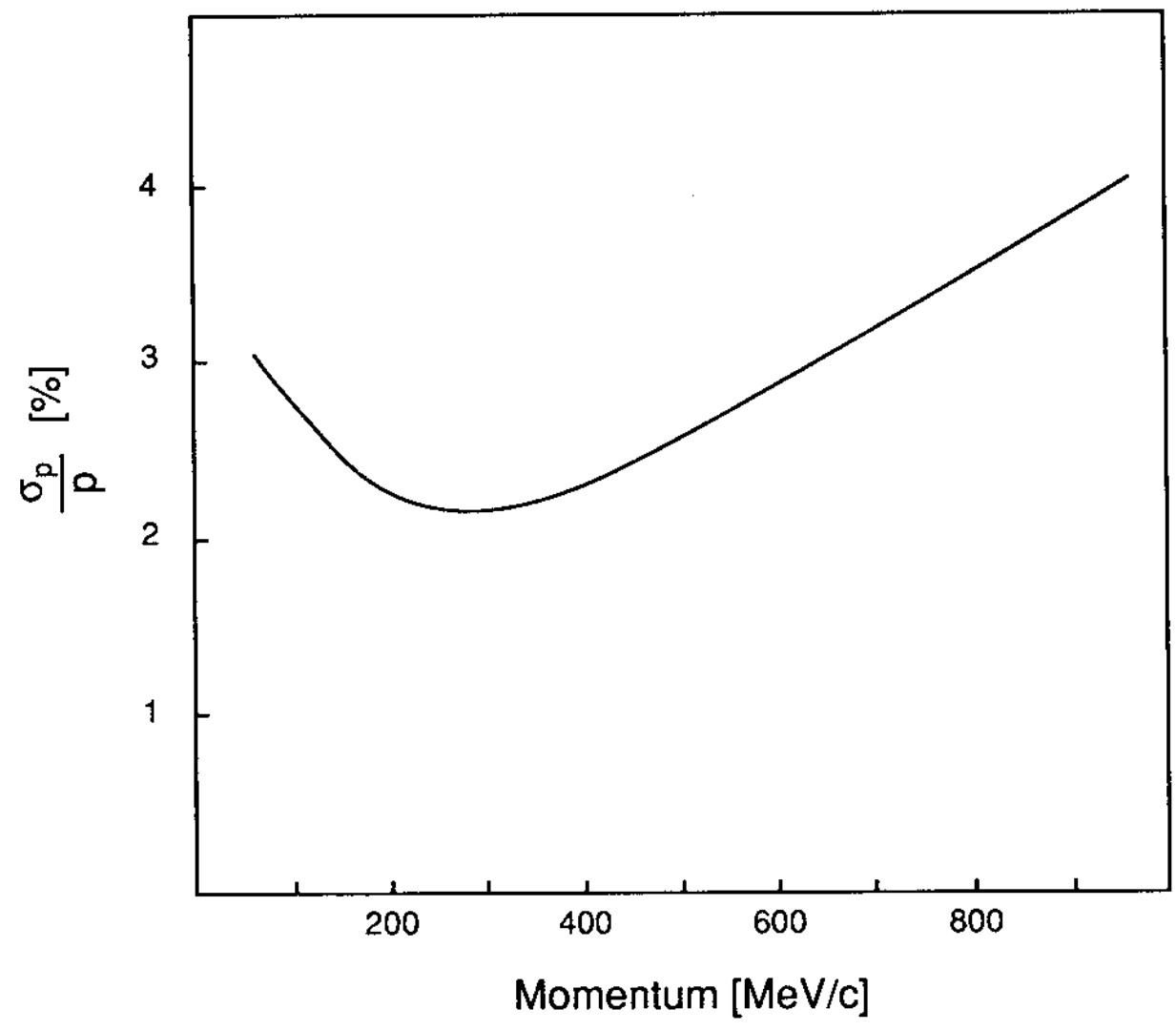

Fig. 18

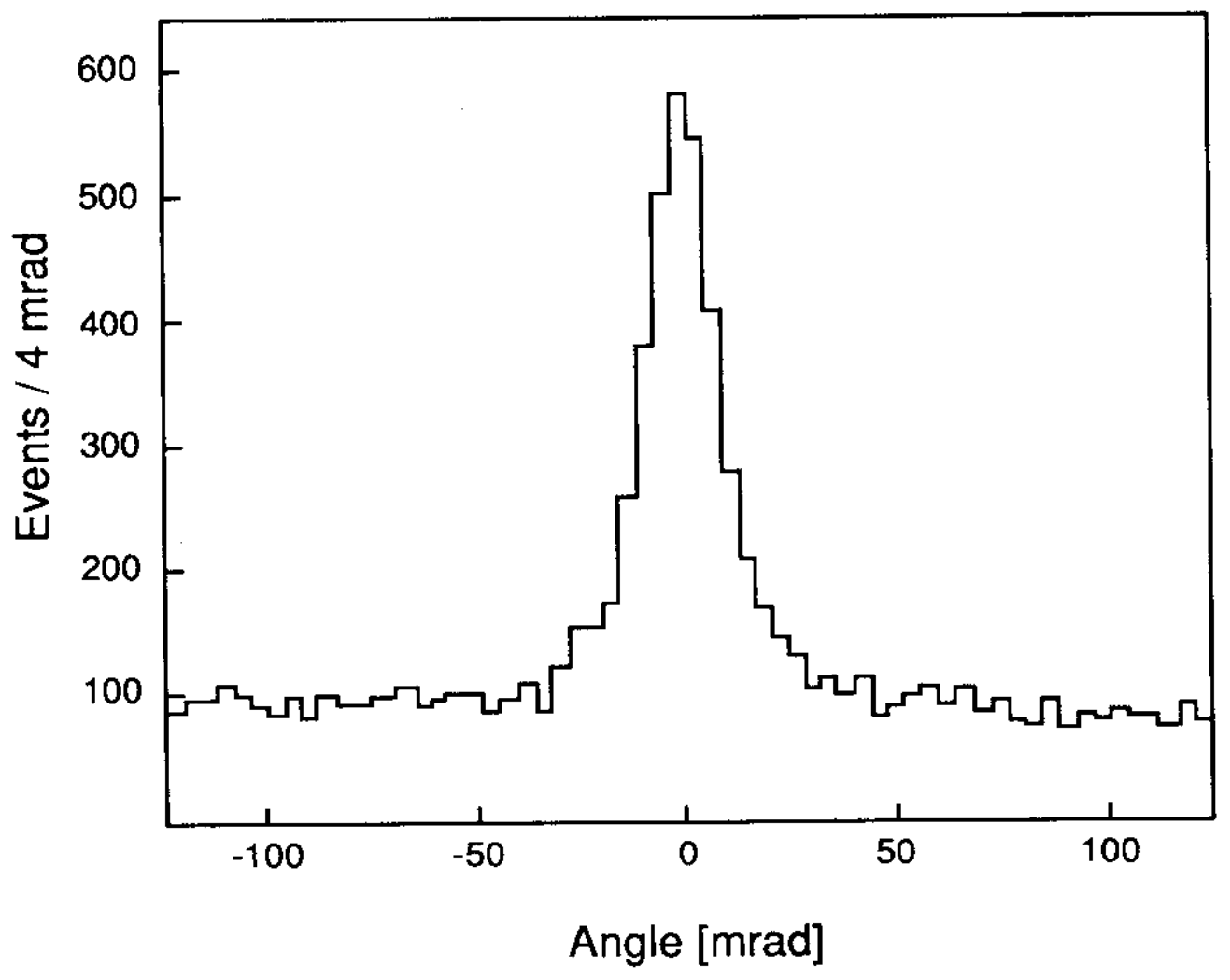

Fig, 19 


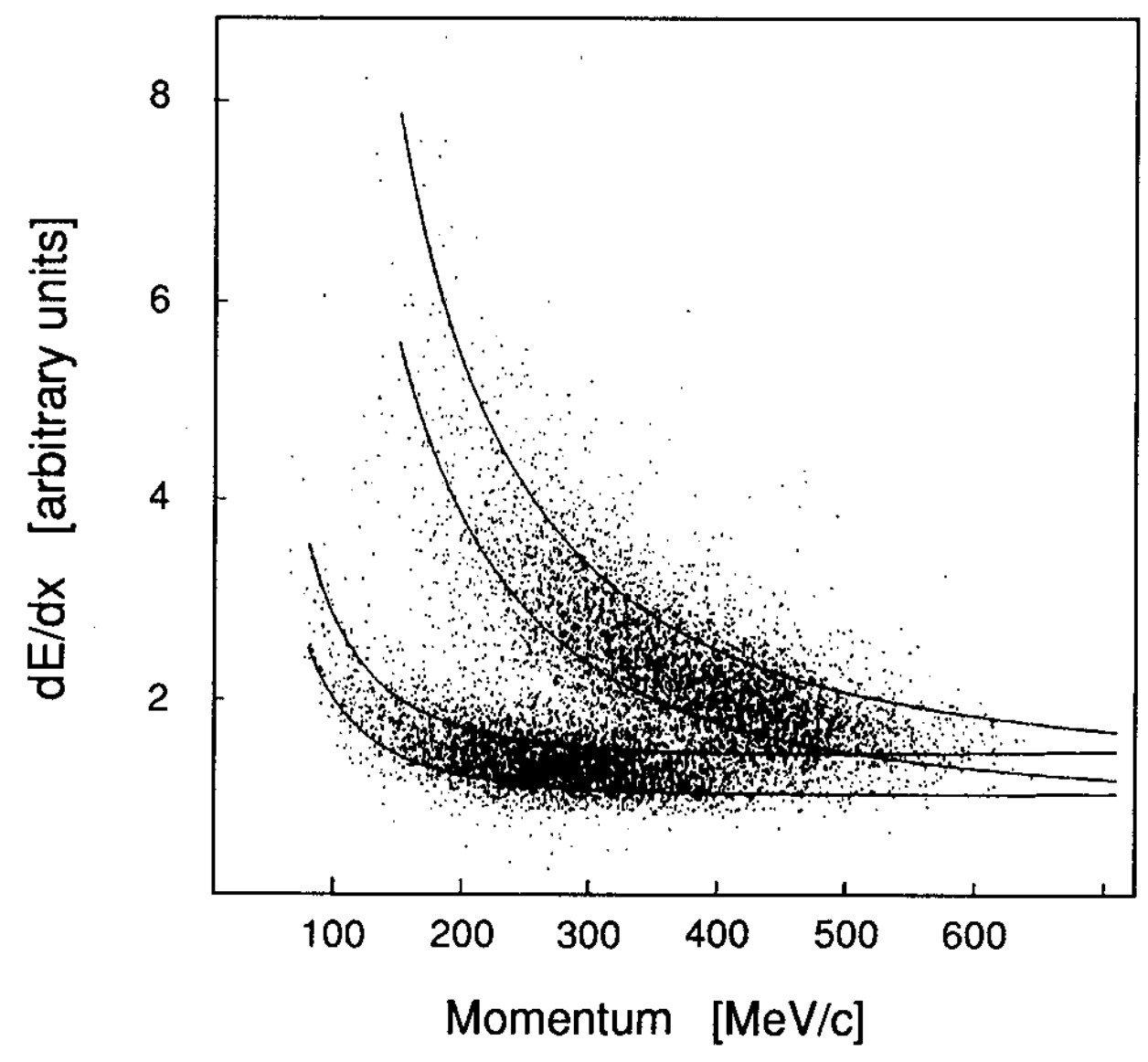

Fig. 20

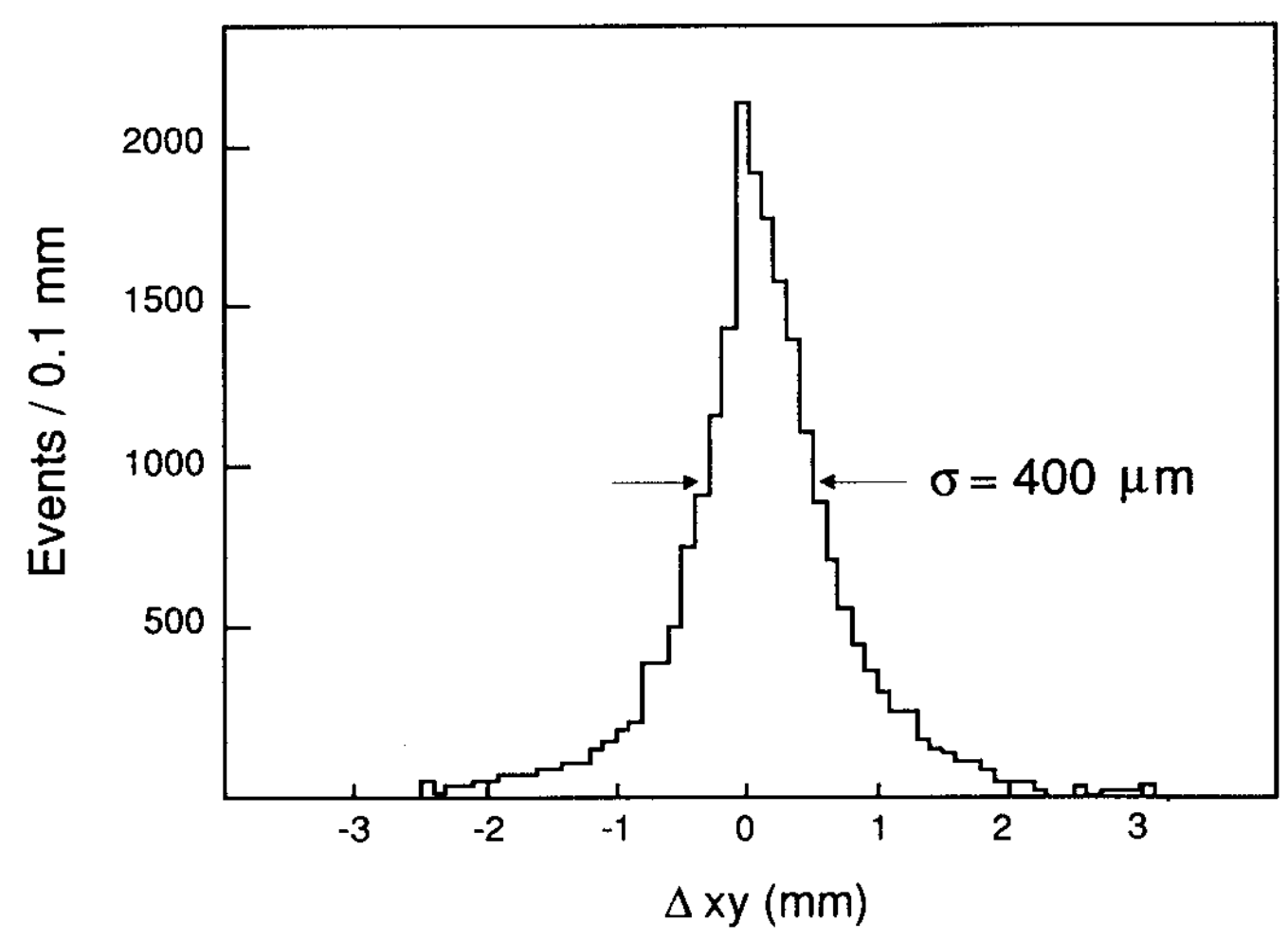

Fig. 21 


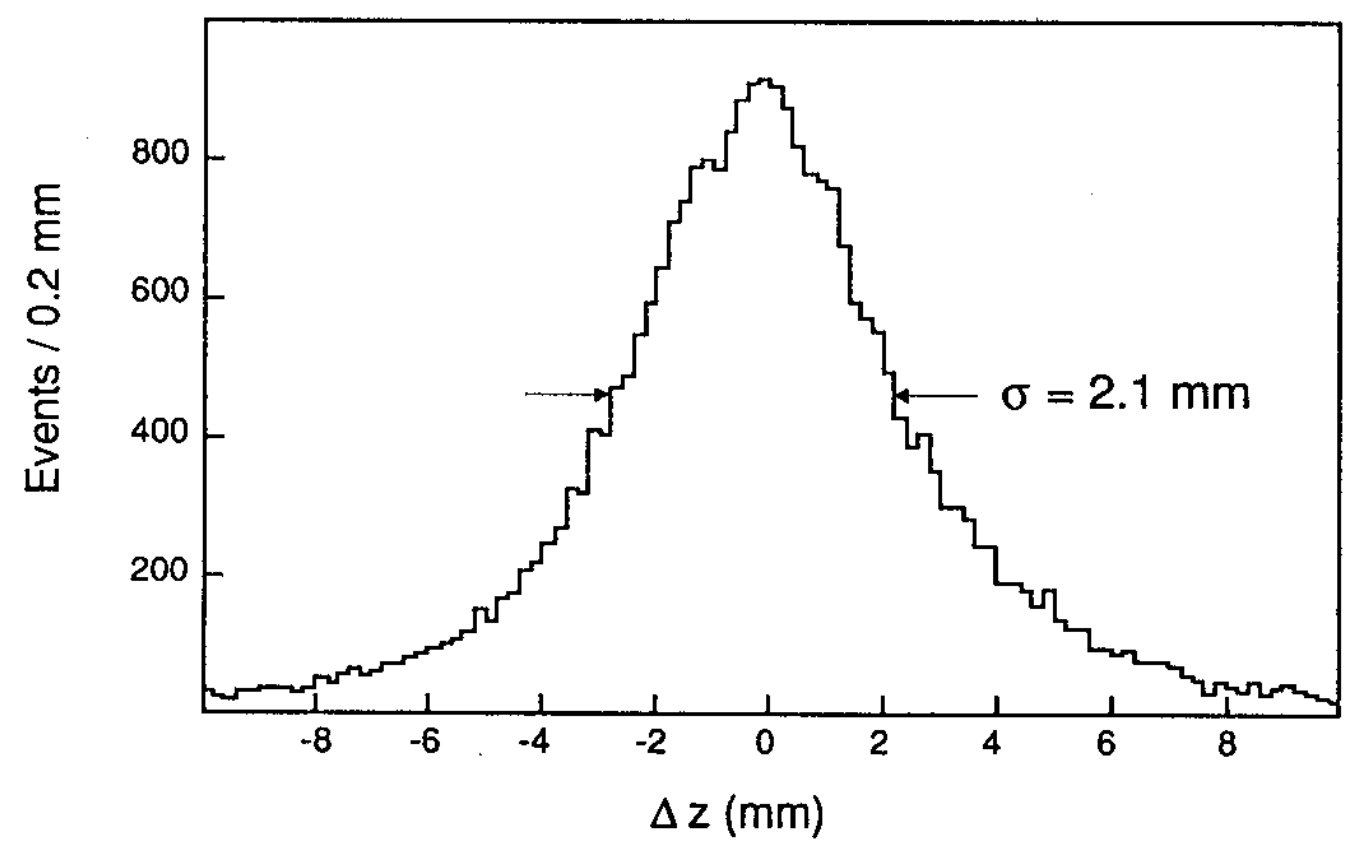

Fig. 22

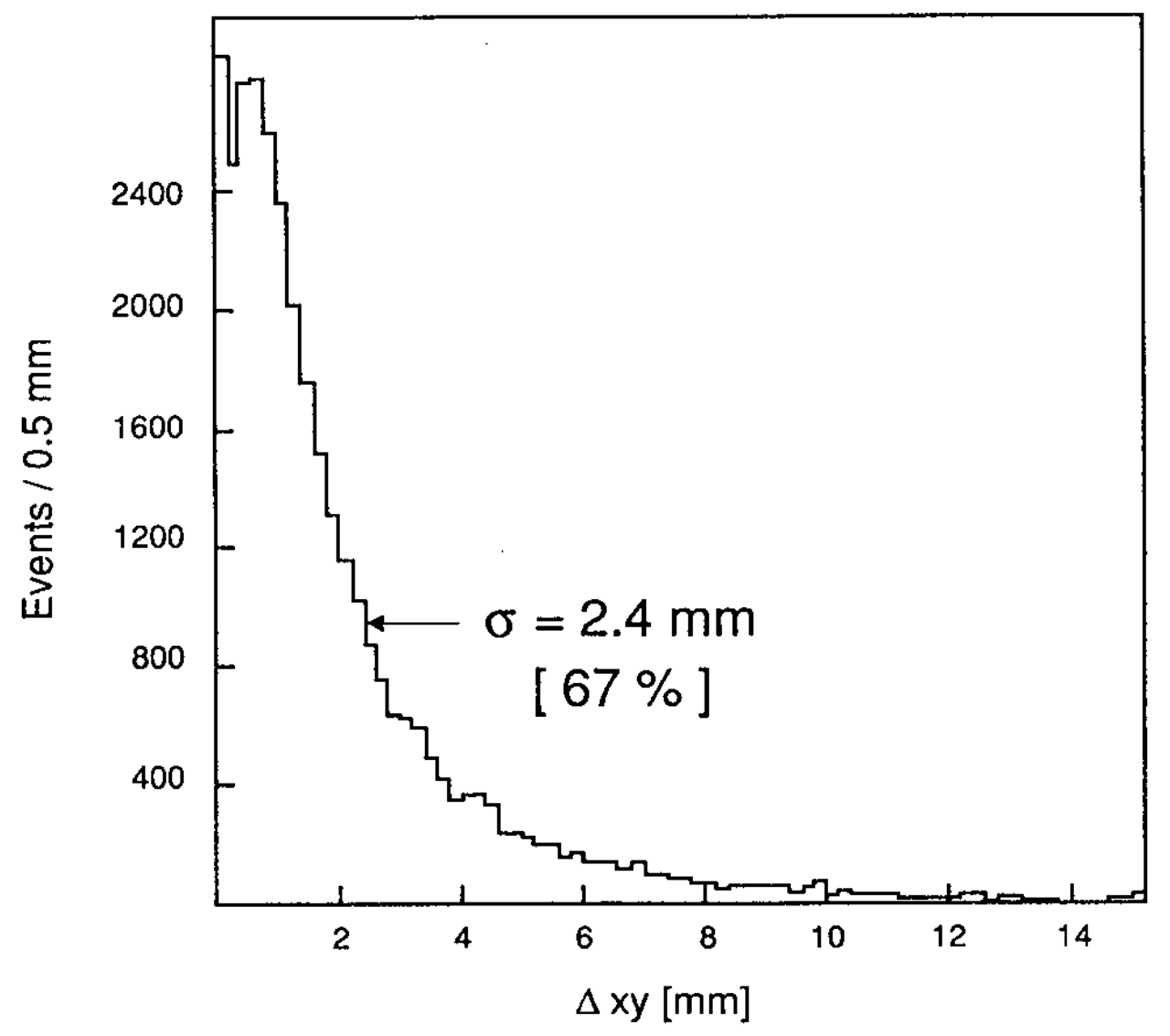

Fig. 23 


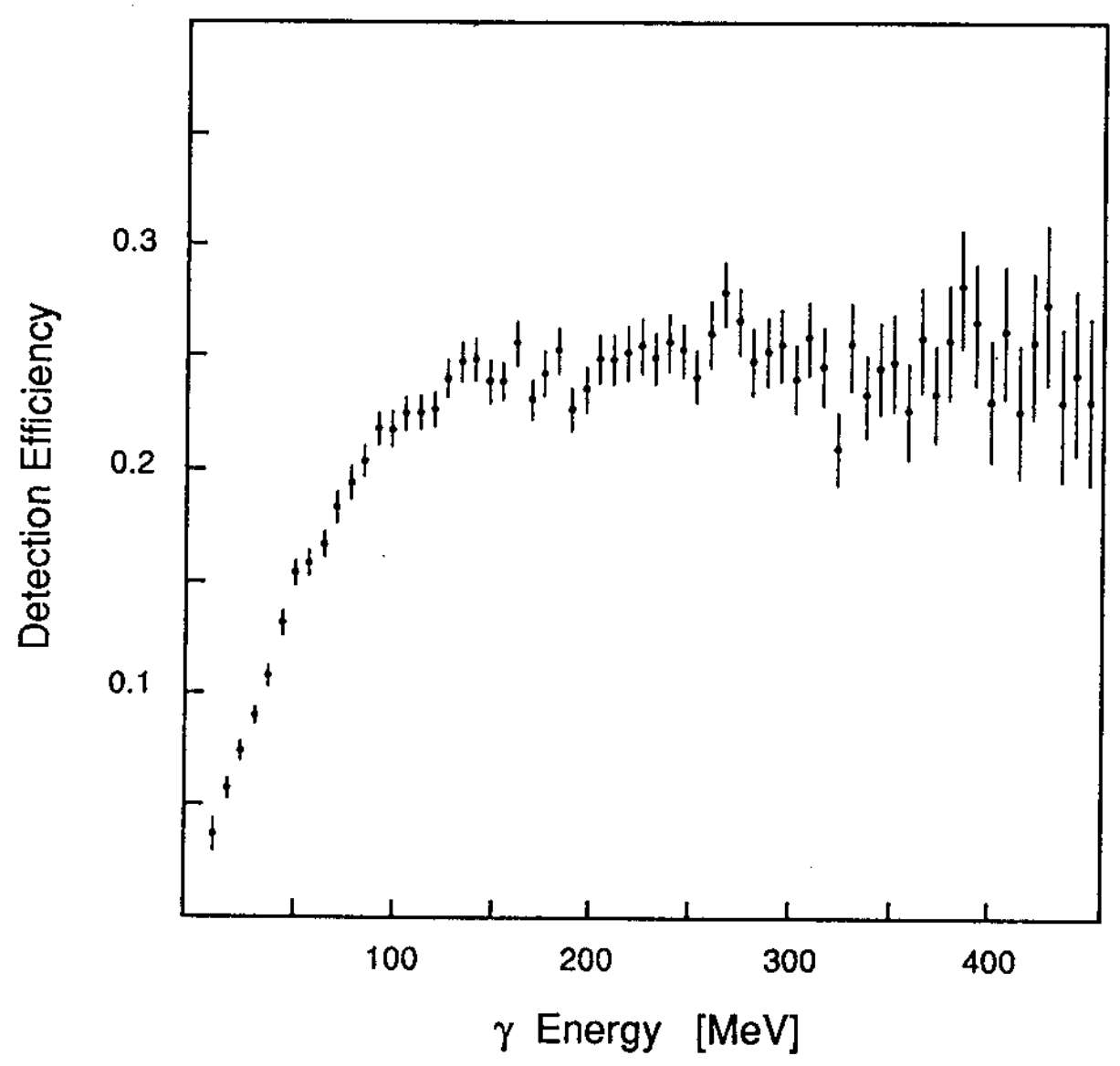

Fig. 24 

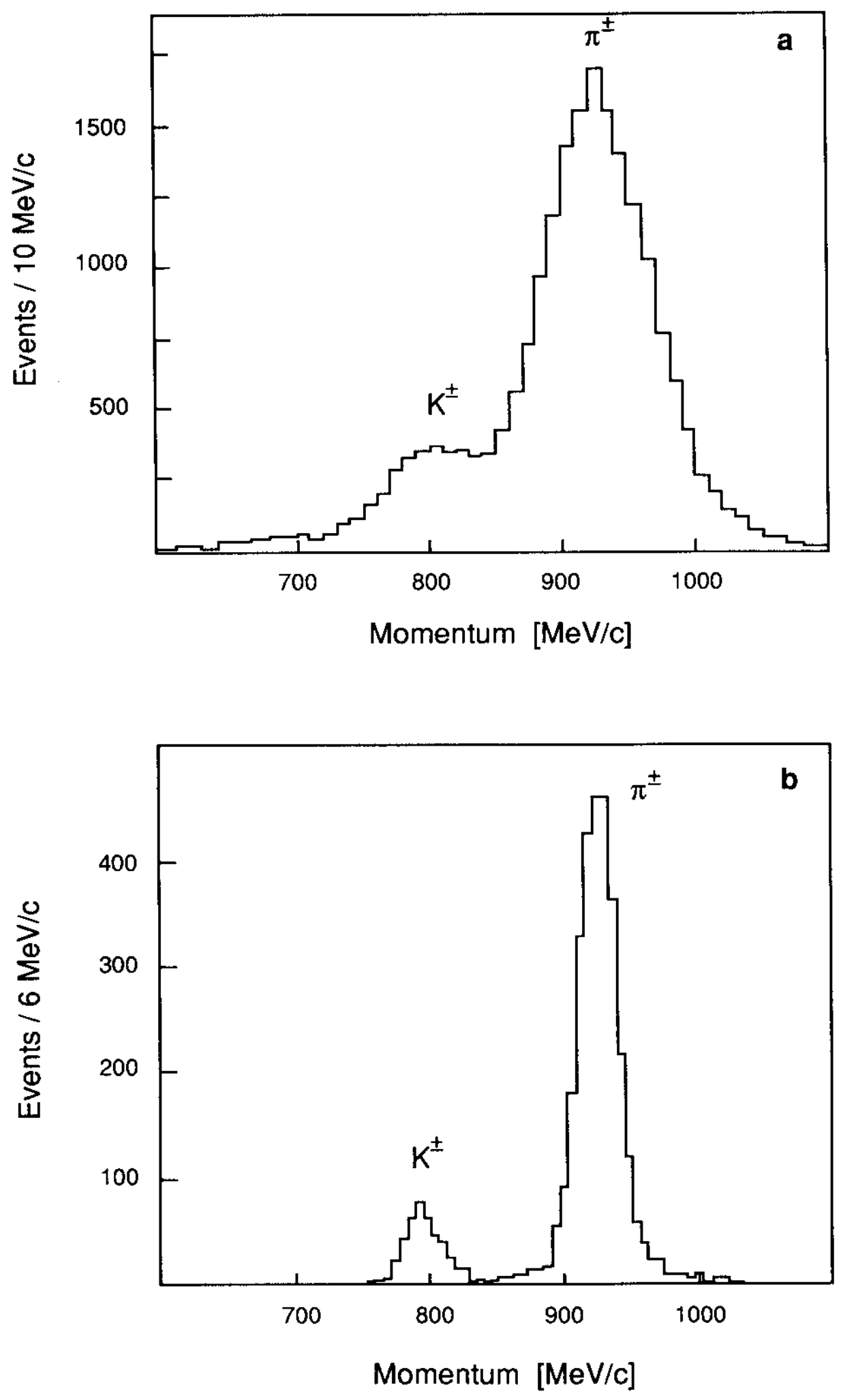

Fig. 25 


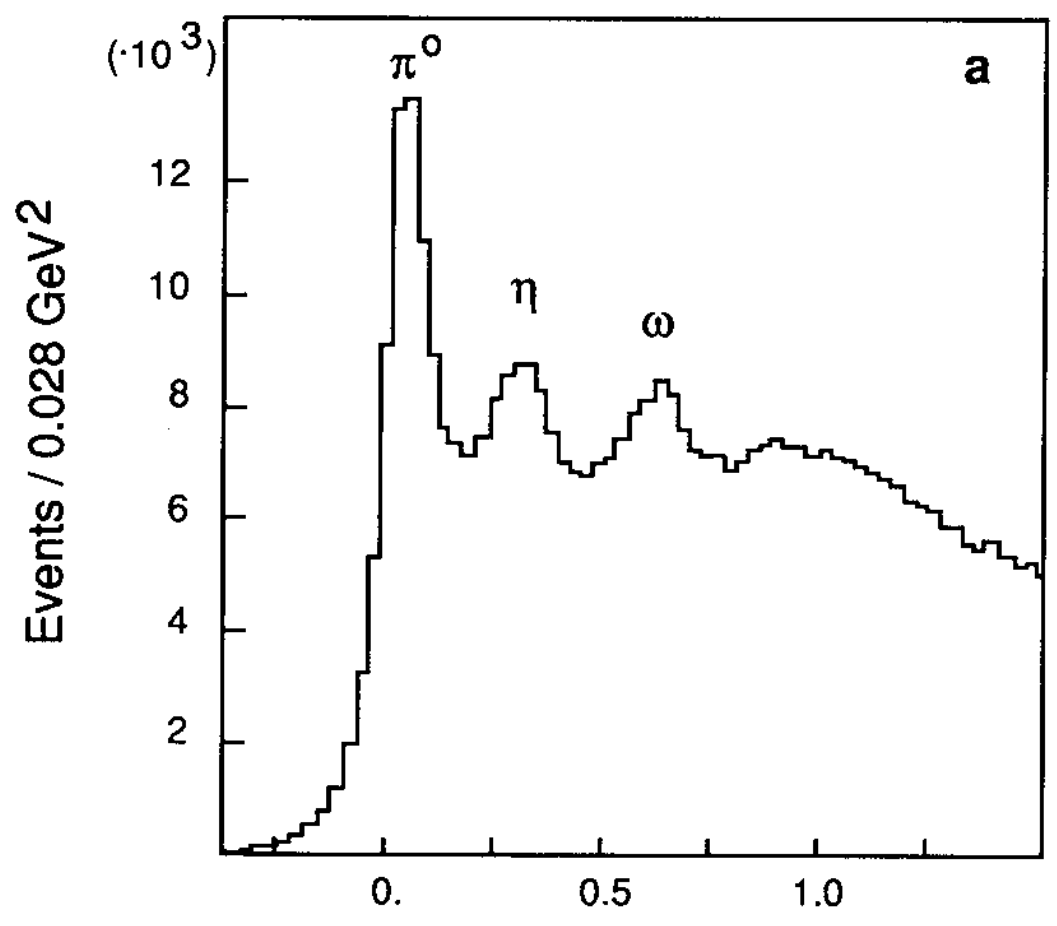

Missing mass squared $\left[\mathrm{GeV}^{2}\right]$

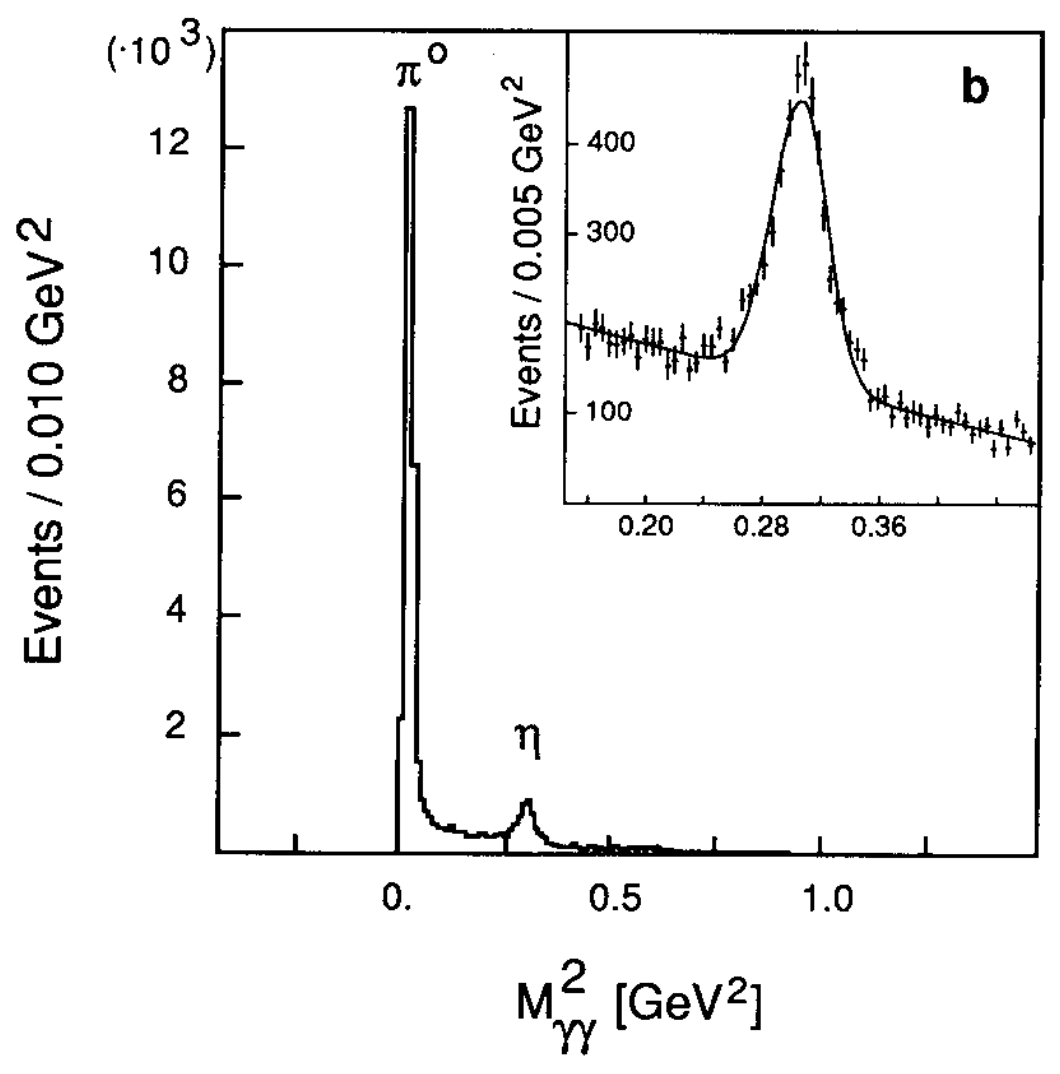

Fig. 26 


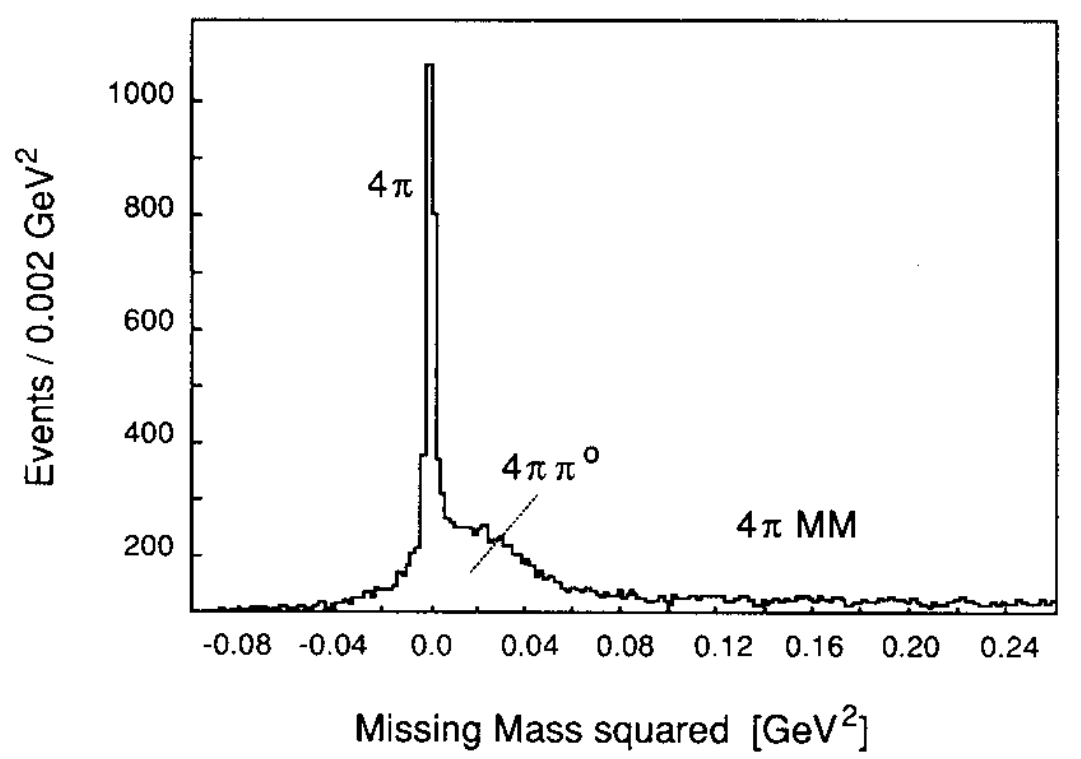

Fig. 27
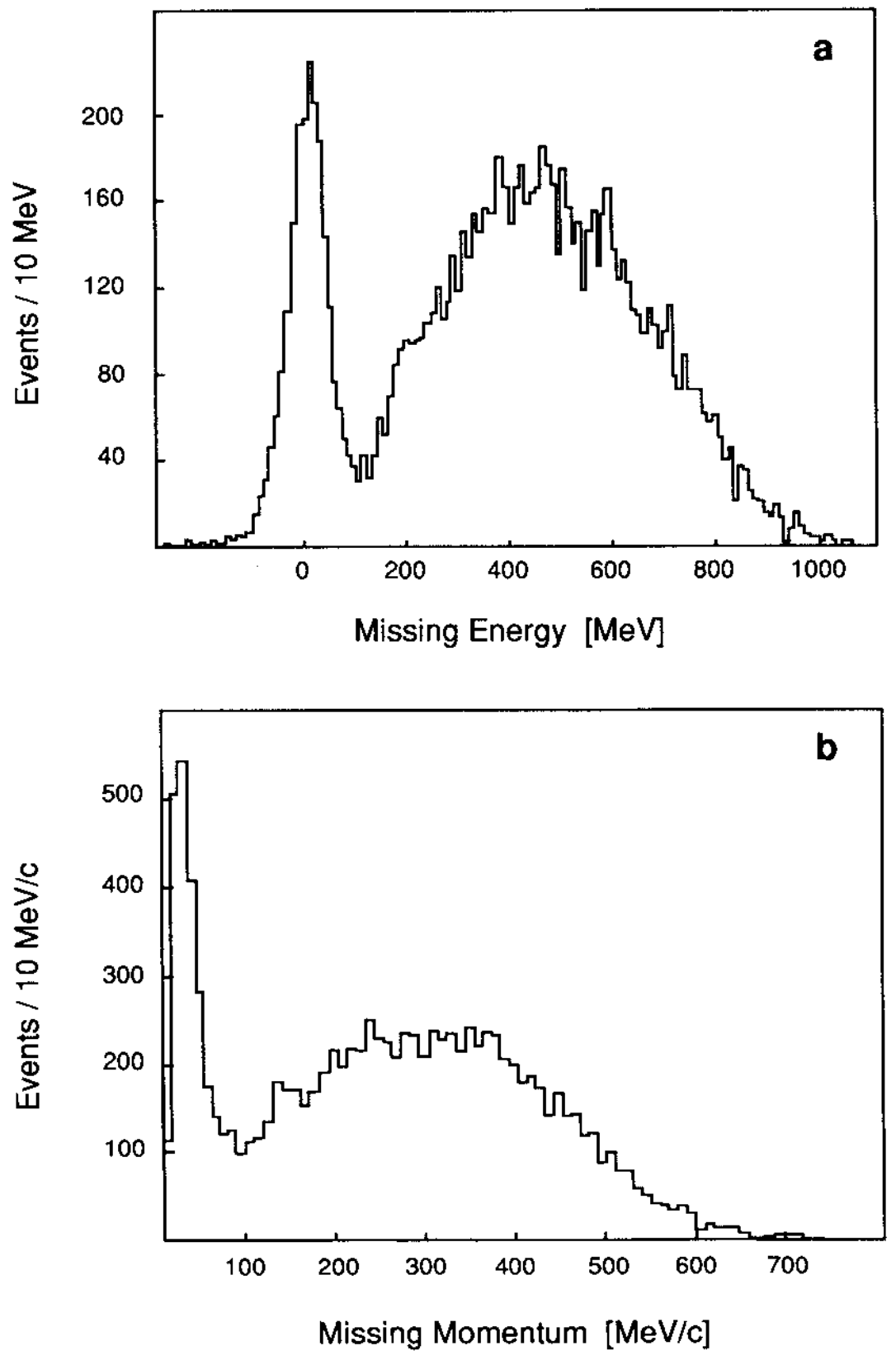

Fig. 28 


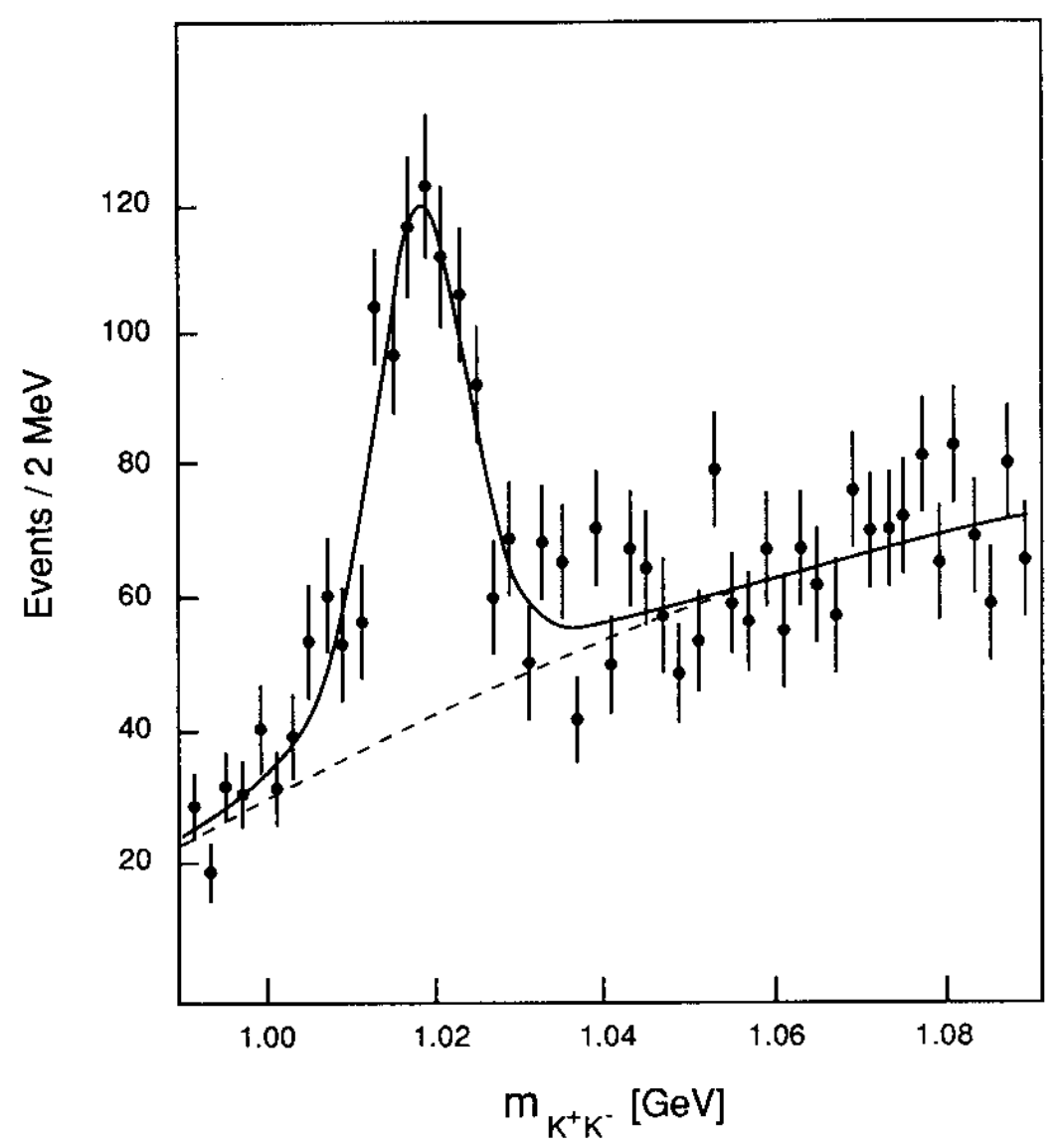

Fig. 29

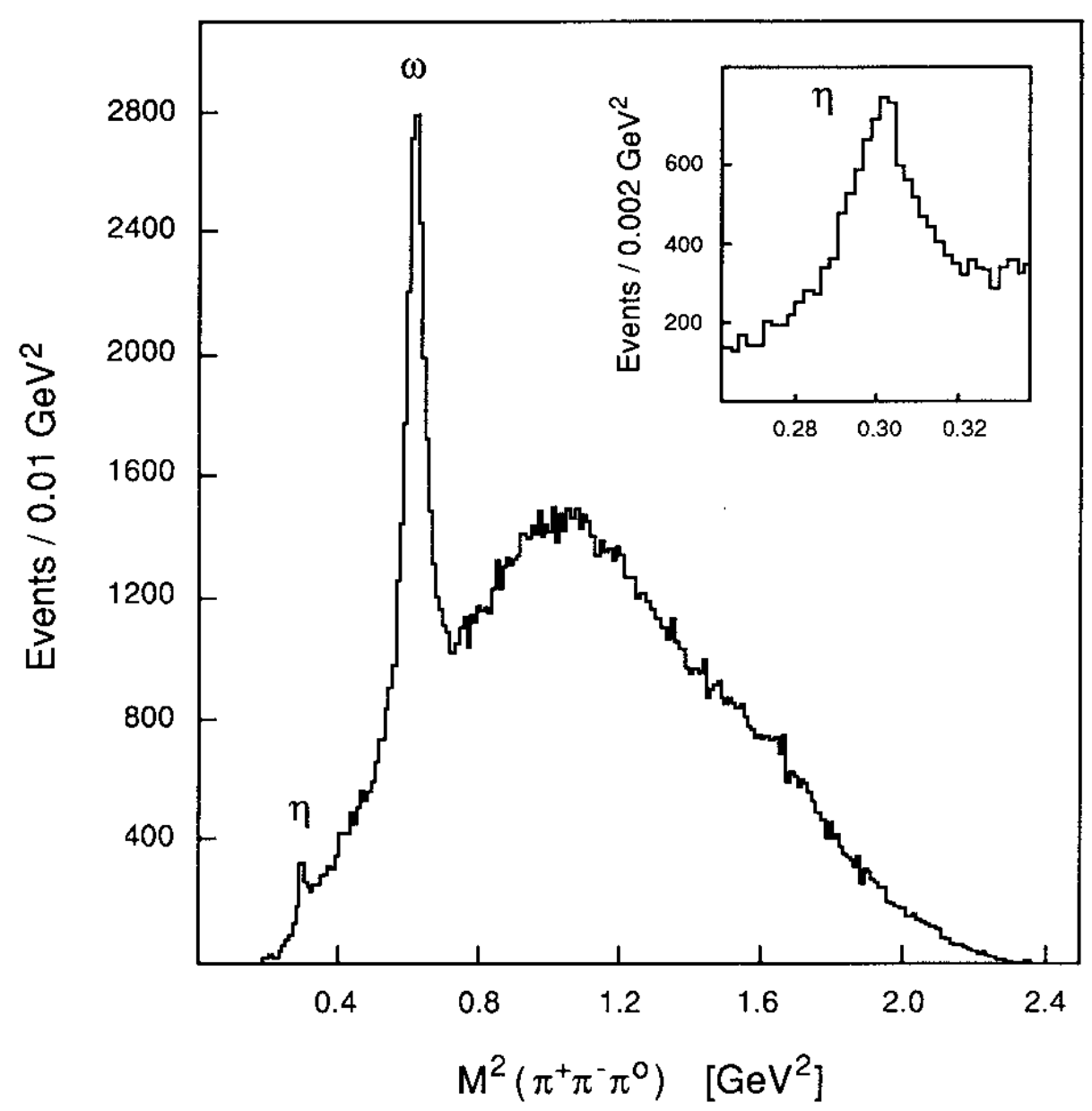

Fig. 30 


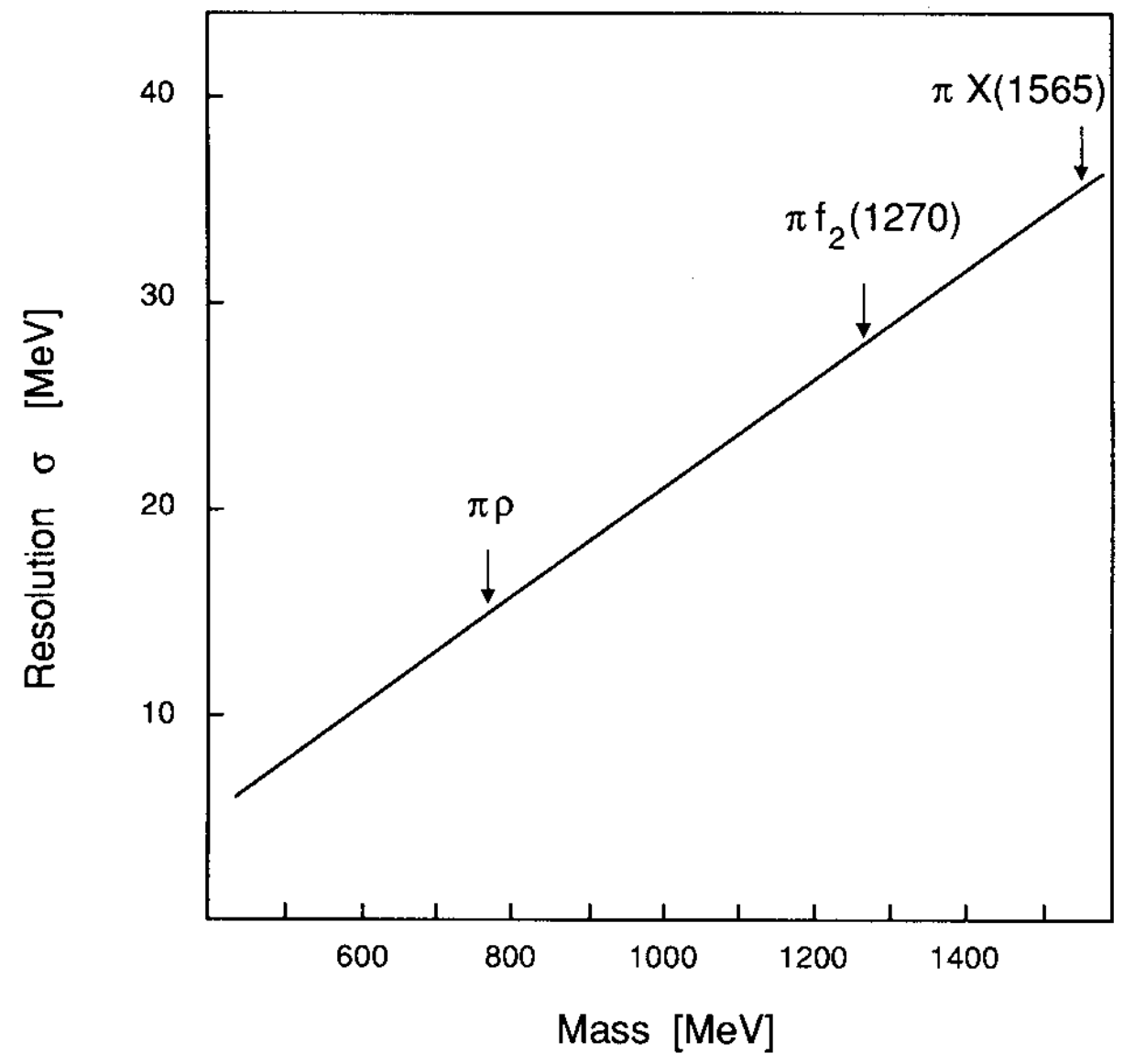

Fig. 37 